\title{
Transcriptome analysis reveals absence of unintended effects in drought-tolerant transgenic plants overexpressing the transcription factor ABF3
}

Ashraf Abdeen ${ }^{1,2 \dagger}$, Jaimie Schnell ${ }^{1,3^{*}+}$, Brian Miki

\begin{abstract}
Background: Plants engineered for abiotic stress tolerance may soon be commercialized. The engineering of these plants typically involves the manipulation of complex multigene networks and may therefore have a greater potential to introduce pleiotropic effects than the simple monogenic traits that currently dominate the plant biotechnology market. While research on unintended effects in transgenic plant systems has been instrumental in demonstrating the substantial equivalence of many transgenic plant systems, it is essential that such analyses be extended to transgenic plants engineered for stress tolerance. Drought-tolerant Arabidopsis thaliana were engineered through overexpression of the transcription factor $A B F 3$ in order to investigate unintended pleiotropic effects. In order to eliminate position effects, the Cre/lox recombination system was used to create control plant lines that contain identical T-DNA insertion sites but with the ABF3 transgene excised. This additionally allowed us to determine if Cre recombinase can cause unintended effects that impact the transcriptome.
\end{abstract}

Results: Microarray analysis of control plant lines that underwent Cre-mediated excision of the ABF3 transgene revealed only two genes that were differentially expressed in more than one plant line, suggesting that the impact of Cre recombinase on the transcriptome was minimal. In the absence of drought stress, overexpression of ABF3 had no effect on the transcriptome, but following drought stress, differences were observed in the gene expression patterns of plants overexpressing ABF3 relative to control plants. Examination of the functional distribution of the differentially expressed genes revealed strong similarity indicating that unintended pathways were not activated.

Conclusions: The action of ABF3 is tightly controlled in Arabidopsis. In the absence of drought stress, ectopic activation of drought response pathways does not occur. In response to drought stress, overexpression of ABF3 results in a reprogramming of the drought response, which is characterized by changes in the timing or strength of expression of some drought response genes, without activating any unexpected gene networks. These results illustrate that important gene networks are highly regulated in Arabidopsis and that engineering stress tolerance may not necessarily cause extensive changes to the transcriptome.

\section{Background}

Drought is a major abiotic stress that limits crop productivity [1]. Climate change models predict an increase in summer drying in the midlatitudes, which could contribute to an increase in the number of episodes of drought $[2,3]$. Engineering plants with

\footnotetext{
* Correspondence: Jaimie.Schnell@inspection.gc.ca

+ Contributed equally

${ }^{1}$ Agriculture and Agri-Food Canada, 960 Carling Ave., Ottawa, ON, K1A 0C6
} Canada

(c) 2010 Abdeen et al; licensee BioMed Central Ltd. This is an Open Access article distributed under the terms of the Creative Commons Attribution License (http://creativecommons.org/licenses/by/2.0), which permits unrestricted use, distribution, and reproduction in any medium, provided the original work is properly cited. enhanced tolerance of abiotic stresses such as drought is a major objective of plant biotechnology that is expected to be commercialized in the near future $[4,5]$. Tolerance to abiotic stress may be achieved through the modification of endogenous plant pathways, often by manipulating important regulatory proteins such as transcription factors. Altering the level of expression of key transcription factors involved in abiotic stress pathways has been shown to enhance tolerance to various abiotic stresses in Arabidopsis [6-9] as well as in 
important crop species such as rice [10-12], maize [13], and alfalfa [14].

Traits involving tolerance to abiotic stresses are considered to be more complex than those that are currently commercialized due to the large number of genes and pathways that may be affected. Furthermore, the interaction between plants and the environment is an intricate, continuous process that has been difficult to characterize, further adding to the complexity of manipulating abiotic stress tolerance traits. The increased complexity of these traits may correspond with a greater potential for unintended effects to occur in transgenic plants.

In transgenic systems, two different types of unintended effects are generally known to occur [15]. Position effects are attributed to the insertion of a transgene at a particular locus in the genome and the resulting interference this might cause. These effects will vary with the site of integration and will therefore be unique to each independent plant line. Position effects can be easily eliminated by screening for plant lines that have no or little position effects. In contrast, pleiotropic effects are independent of the site of transgene insertion and are the sum of all the phenotypic effects caused by expression of the transgene. While some of these may be the intended trait, others may occur through unexpected interactions of the gene with plant processes and constitute the unintended pleiotropic effects. These effects are of greater interest since they are more difficult to eliminate and more likely to create safety issues.

Engineering more complex traits such as abiotic stress tolerance in plants through the manipulation of transcription factors may uncover cryptic properties of the transcription factor that could produce some of the unintended pleiotropic effects. Many transcription factors are part of large families that have complex evolutionary histories $[16,17]$. These families typically arise through gene duplications followed by functional divergence in separate expression domains or through the acquisition of new functions. These processes often result in functional redundancies within the families that can be difficult to detect. Furthermore, some transcription factors may retain ancestral functions that are sometimes only revealed by altering the normal pattern of expression. Therefore the manipulation of transcription factors in engineering complex traits such as abiotic stress tolerance may be likely to produce unintended pleiotropic effects.

The use of non-targeted global profiling technologies, such as microarray analysis, to identify unintended effects in plant systems has proven an effective means of determining the "substantial equivalence" of a transgenic plant to its non-transgenic counterpart. Such approaches have been used to investigate unintended effects in a number of transgenic plant systems [18-27]. To date, these studies have primarily focused on simple, monogenic traits such as those that are currently commercially grown. As transgenic crops with more complex traits involving the modification of endogenous plant pathways will soon be entering the market, it is important to extend these analyses to investigate the potential for unintended pleiotropic effects in such systems.

In order to understand the extent and kinds of unintended effects that could be induced in transgenic plants engineered for complex traits, we conferred drought tolerance on Arabidopsis thaliana by overexpressing the transcription factor $A B F 3$. This system targets drought resistance, a trait that will likely enter the market in the near future. Since transcription factors ultimately function by altering the levels of expression of target genes, we investigated unintended effects using microarray analysis to survey global gene expression profiles. In order to eliminate position effects in our analysis and focus on the pleiotropic unintended effects, we employed the Cre/lox system to excise the $A B F 3$ transgene from the site of insertion, leaving behind the selectable marker, to create control plant lines. Without the ABF3 transgene, the pleiotropic effects will be absent but the site of integration is still interrupted by the selectable marker such that position effects are maintained in these lines.

ABF3 belongs to the ABF/AREB subfamily of bZIP transcription factors which consists of thirteen members in Arabidopsis. Several members have been shown to function in ABA signalling either during seed maturation or in response to stress [28]. These factors can bind to ABA-response elements (ABREs), cis-regulatory elements found in the promoters of many ABA- and stress-responsive genes [29-31]. In addition to drought tolerance, overexpression of $A B F 3$ confers tolerance to salt, cold, heat, and oxidative stresses, suggesting that it regulates multiple abiotic stress pathways in Arabidopsis $[7,32]$. Three other ABF/AREB transcription factors are predicted to function in ABA-dependent stress signalling based on expression profiling and overexpression studies. Expression of $A B F 1$ is induced by cold-treatment [29]. $A B F 2 / A R E B 1$ is induced by salt-treatment as well as dehydration but not cold and overexpression confers tolerance to a wide range of abiotic stresses, including salt, drought, heat, and oxidative stress [29,31,33]. Interestingly, $A B F 2 / A R E B 1$ also appears to function in glucose signalling as well as in the regulation of seedling growth [33]. ABF4/AREB2 is expressed in response to cold, drought, and salt and overexpression renders plants tolerant to drought and salt $[7,29,31]$. Therefore, ABF3 likely shares some redundant functions with other members of the ABF/AREB subfamily.

Plant response to drought involves changes in the expression patterns of a large number of genes [34-36] 
and, in addition to members of the ABF/AREB family, a number of other transcription factors have been identified that play a role in the drought response in Arabidopsis. These include the AP2/ERF transcription factors DREB2A, DREB2B, and CBF4 [8,37,38], AtMYB2, which functions in concert with AtMYC2 [39-41], and the NAC family transcription factors ANAC19, ANAC055/ ATNAC3, and ANAC072/RD26 [42], which at least partially function in concert with a zinc finger homeodomain protein ZFHD1 [43]. Therefore, while overexpression of ABF3 affects one of the key drought response pathways, it is not the only pathway mediating the drought response at the gene expression level.

The use of the Cre/lox system to create control lines also created an opportunity to examine the effects of the Cre/lox system on the transcriptome. Site-specific recombination technologies can be used to excise selectable markers or other undesirable genetic elements and can also be used to direct site-specific integration of transgenes [44]. While many studies have employed Cre-mediated recombination in plant systems with no apparent unintended effects [45-48], other studies have observed a range of abnormal phenotypes including growth defects, leaf chlorosis, delayed flowering, and male sterility $[49,50]$. In tobacco plants transformed with a chloroplast targeted Cre recombinase, recombination was observed involving cryptic lox sites in the plastid genome, but invariably the second lox site was located within the transgene [51-53]. These recombinations could result in deletions of up to $147 \mathrm{~kb}$, but they did not cause any deleterious effects in the plants [51-53]. Studies in animal systems have similarly revealed that Cre recombinase can have unintended effects, often leading to chromosomal aberrations [54-57].

These studies suggest that the Cre/lox system has the potential to cause unintended effects in plant systems, by mediating recombination with cryptic lox sites that may be present in the genome resulting in large deletions. Such cryptic lox sites are difficult to identify since they may deviate substantially from conventional loxP sites [52] and not all of the unintended effects may produce readily apparent phenotypic abnormalities, so studying the unintended effects of Cre recombinase using a non-targeted approach such as microarray analysis is essential for establishing the utility and safety of this technology.

In this study, we performed microarray analysis on Arabidopsis plants engineered to be drought-tolerant through overexpression of the transcription factor $A B F 3$ with the goal of identifying unintended pleiotropic effects. The results suggest that overexpression of $A B F 3$ has a minimal impact on the transcriptome, with differences in the gene expression pattern only detectable in response to drought and then being suggestive of transcriptional reprogramming as opposed to the activation of novel pathways. In addition, we examined the impact of Cre recombinase on the transcriptome to detect any unintended effects of this technology and found that it had minimal effects on gene expression patterns in plants following transgene excision.

\section{Results}

\section{Phenotype of plants overexpressing ABF3}

A construct was created containing the CaMV $35 \mathrm{~S}$ promoter followed by the coding sequence of $A B F 3$ and then the nopaline synthase transcriptional terminator, all of which was flanked by two loxP sites (Figure 1A). This construct was transformed into Arabidopsis thaliana to generate 35S:ABF3 plants. Fifty-nine independent transformants were recovered and three high-expressing lines containing single insertions were selected for further analysis (35S:ABF3-48, -57, and -59).

Control plant lines were generated by excising the 35S:ABF3 transgene, leaving only the selectable marker transgene at the site of insertion (Figure 1B). This was

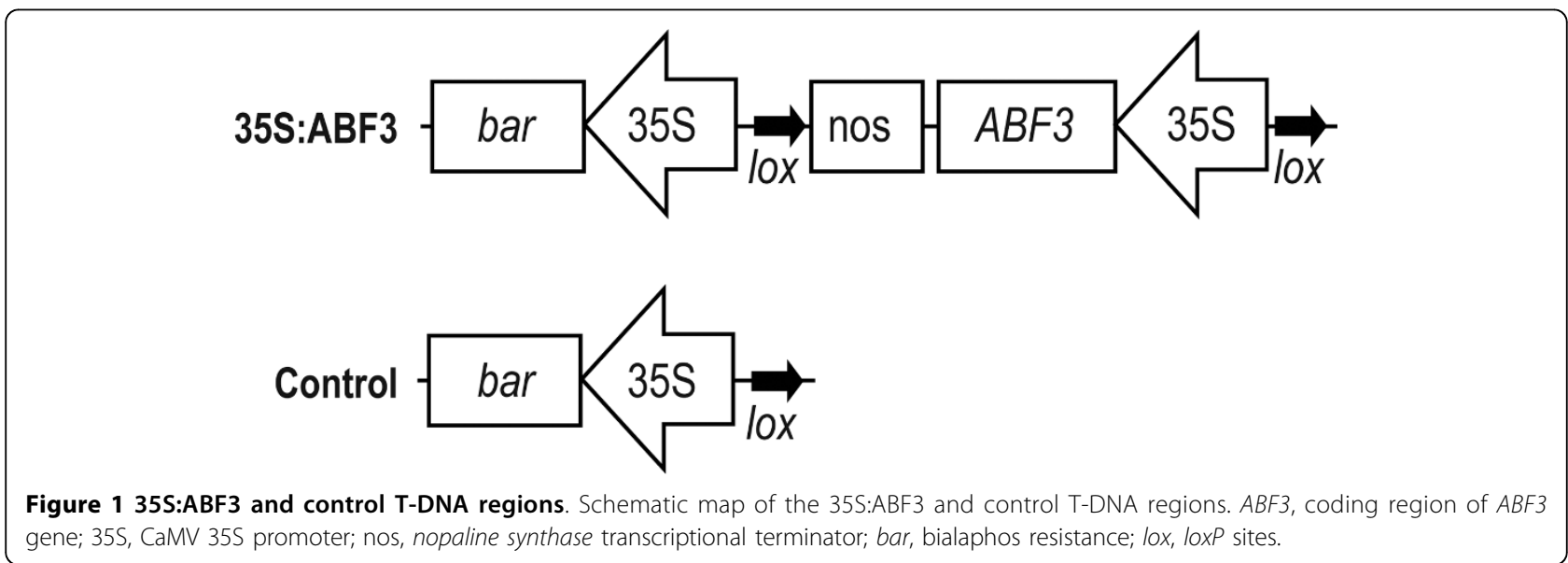


achieved by crossing 35S:ABF3 plants with plants expressing the Cre recombinase gene and then backcrossing the progeny to wild-type Arabidopsis to remove the Cre recombinase gene, generating three control lines (Control-48, -57, and -59). The loss of both the ABF3 transgene and the Cre recombinase transgene was confirmed by PCR (Additional file 1).

Previously, Arabidopsis plants overexpressing ABF3 were found to be more tolerant to drought conditions, which could at least partially be attributed to a lower rate of transpiration [7]. To confirm that the 35S:ABF3 lines show a similar phenotype, the transpiration rate was determined. Excised leaves from 4-week-old 35S: ABF3 plants left at room temperature for 1 day lost less fresh weight than both wild-type and control plants, indicating a lower rate of transpiration (Figure 2A). In addition, 35S:ABF3 plants showed a mild growth retardation that became more evident as plants matured

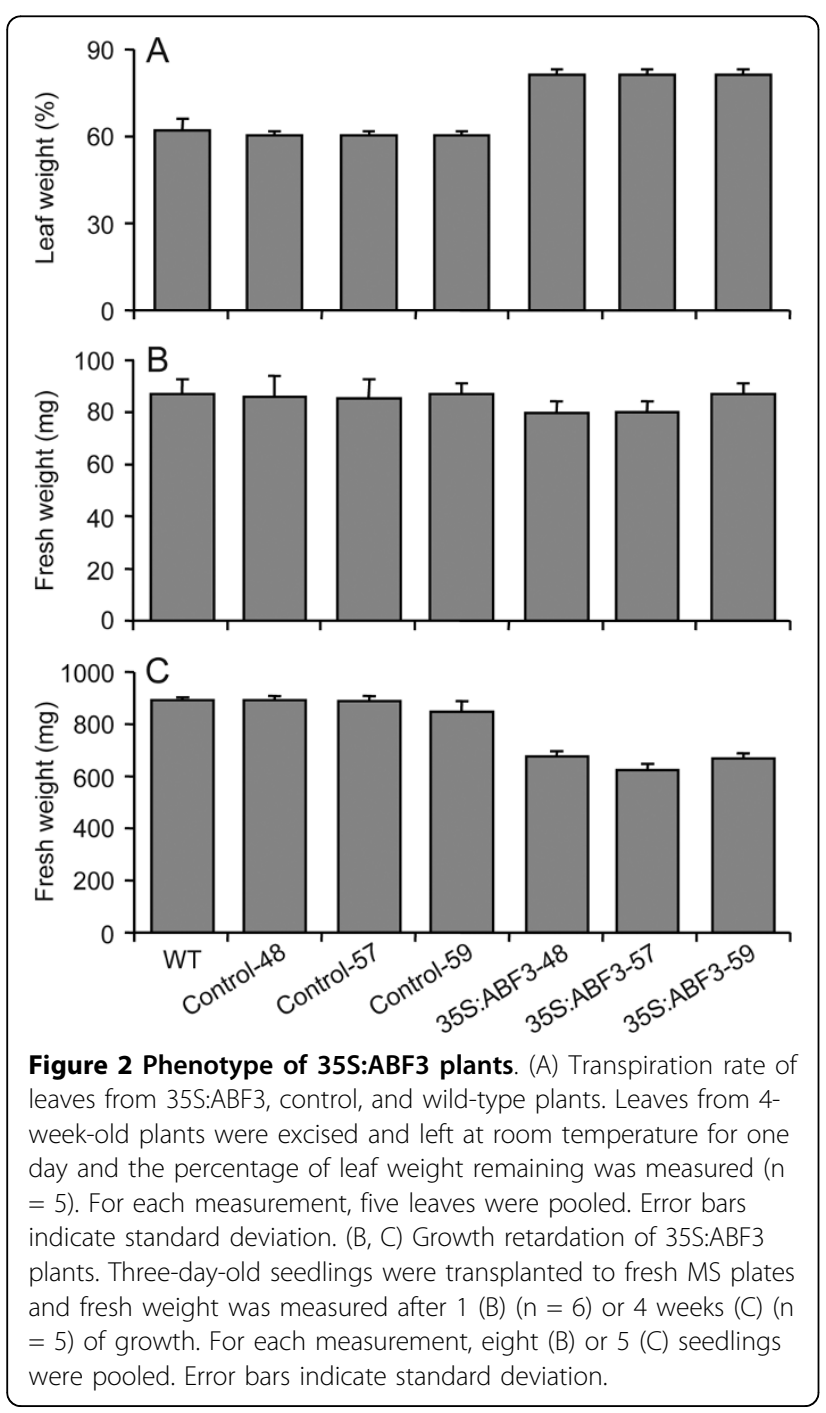

(Figure 2B, C), which is also consistent with previous results [7].

\section{Identification of position effects and impact of Cre recombinase on the transcriptome}

Microarray analysis of control plant lines was performed to identify position effects as well as to determine if use of the Cre/lox system can cause unintended effects in plant systems. Position effects should be specific to each of the three control lines, as each line is expected to have a unique insertion site. In contrast, unintended effects resulting from excision at cryptic lox sites are more likely to affect independent plant lines similarly, since cryptic sites that may be present in the Arabidopsis genome will equally be found in each plant line.

Using a $P$-value cut-off of 0.05 , only a small number of genes were differentially expressed in control lines compared to wild-type plants (Table 1). In the Control48 line, only a single gene was differentially expressed. In the Control-57 and -59 lines, 10 and 4 genes were differentially expressed and two of these, rps 7 (AtCg00900/AtCg01240) and rps12.1 (transplice part 1 of 2) (AtCg00065), were common to both lines. These genes could represent either position effects or natural background variation in gene expression. The rps 7 and rps12.1 genes that were differentially expressed in both Control-57 and -59 lines may reflect unintended effects of the Cre/lox system.

\section{Effect of $A B F 3$ overexpression on the transcriptome in the absence of drought}

Microarray analysis of 35S:ABF3 plants was performed to identify unintended effects resulting from overexpression of a transcription factor in the absence of stress. Expression of $A B F 3$ in the absence of stress is generally very low but it is rapidly induced in response to $A B A$ $[7,29]$. Overexpression of $A B F 3$ would therefore be expected to initiate those pathways that are typically activated in response to stress via ABA-mediated signalling. This has been observed for other transcription factors and is generally used as a means of identifying targets of that particular transcription factor $[39,42,58]$.

Three independent plant lines containing the 35S: ABF3 transgene were compared to the corresponding control plant lines from which the 35S:ABF3 transgene was excised. Based on microarray analysis, using a cutoff of $P<0.05$, only a small number of genes $(7,1$, and 8 ) were differentially expressed in the three $35 \mathrm{~S}: \mathrm{ABF} 3$ transgenic plant lines (Table 2). The only gene that was differentially expressed in all three plant lines was $A B F 3$.

\section{Drought response of 35S:ABF3 plants}

Overexpression of $A B F 3$ in Arabidopsis confers enhanced drought tolerance [7]. Since ABF3 is a 
Table 1 Genes differentially expressed $(P<0.05)$ in control lines following Cre-mediated excision of the $A B F 3$ transgene

\begin{tabular}{|c|c|c|c|c|c|}
\hline Line & Probe Set ID & AGI & Annotation & M & $\mathbf{P}$ \\
\hline 48 & 267442_at & At2g19080 & Metaxin-related & 0.68 & $1.22 \times 10^{-2}$ \\
\hline \multirow{11}{*}{57} & 244939_at & AtCg00065 & Ribosomal protein s12 (Transplice part 1 of 2) (rps12.1)* & 2.58 & $9.11 \times 10^{-4}$ \\
\hline & 244992_s_at & AtCg00900/AtCg01240 & Ribosomal protein s7 (rps7)* & 1.50 & $2.97 \times 10^{-2}$ \\
\hline & 245608_at & At4g14350 & Protein kinase family protein & -0.99 & $5.86 \times 10^{-3}$ \\
\hline & 247137_at & At5g66210 & Calcium-dependent protein kinase family protein (CDPK28) & -1.20 & $3.35 \times 10^{-2}$ \\
\hline & 247754_at & At5g59080 & Expressed protein & 1.33 & $2.20 \times 10^{-2}$ \\
\hline & 248812_at & At5g47330 & Palmitoyl protein thioesterase family protein & 2.34 & $7.24 \times 10^{-3}$ \\
\hline & 252053_at & At3g52400 & Syntaxin, putative (SYP122) & -1.09 & $3.60 \times 10^{-2}$ \\
\hline & 255655_at & At4g00980 & Zinc knuckle (CCHC-type) family protein & 1.39 & $4.60 \times 10^{-6}$ \\
\hline & 259072_at & At3g11700 & Fasciclin-like arabinogalactin protein 18 precursor (FLA18) & -0.73 & $2.29 \times 10^{-3}$ \\
\hline & 259348_at & At3g03770 & Leucine-rich repeat transmembrane protein kinase, putative & 0.91 & $1.82 \times 10^{-2}$ \\
\hline & 244939_at & AtCg00065 & Ribosomal protein s12 (Transplice part 1 of 2) (rps12.1)* & 2.50 & $1.52 \times 10^{-3}$ \\
\hline \multirow[t]{3}{*}{59} & 244992_s_at & AtCg00900/AtCg01240 & Ribosomal protein s7 (rps7)* & 1.51 & $2.69 \times 10^{-2}$ \\
\hline & 246727_at & At5g28010 & Bet $v$ I allergen family protein & 2.02 & $1.10 \times 10^{-5}$ \\
\hline & 262719_at & At1g43590 & Transposable element gene & 1.29 & $1.30 \times 10^{-2}$ \\
\hline
\end{tabular}

$M$ is log2 fold change and $P$ is FDR-adjusted $P$-value for Student's $t$-test.

* indicates genes encoded by the chloroplast genome.

transcription factor, it is likely that this is achieved at the level of gene expression. Therefore, microarray analysis should provide insight into the mechanism of drought tolerance. Since the expression pattern of $A B F 3$ during the drought response is achieved by constitutive overexpression from the CaMV $35 \mathrm{~S}$ promoter, it is possible that unintended effects may also be generated downstream. By comparing the transcriptional profile of 35S:ABF3 plants to control plants, it may be possible to identify these unintended effects. Two time points were examined in order to consider both early and late responses to drought. For this analysis, the Control-48 and 35S:ABF3-48 lines were selected as the Control-48 lines showed the smallest number of differentially expressed genes (Table 1).

Following $2 \mathrm{~h}$ of drought stress, using a cut-off of $P<$ 0.05 and a fold-change of 2, 508 genes were differentially expressed in the control plants exposed to drought stress compared to unstressed control plants and 468 genes were differentially expressed in 35S:ABF3 plants exposed to drought stress compared to unstressed 35S: ABF3 plants. Following $24 \mathrm{~h}$ of drought stress, 592

Table 2 Genes differentially expressed $(P<0.05)$ in 35S:ABF3 transgenic plants

\begin{tabular}{|c|c|c|c|c|c|}
\hline Line & Probe Set ID & AGI & Annotation & M & $\mathbf{P}$ \\
\hline & 244964_at & AtCg00580 & PSII cytochrome b559 (psbE)* & 0.73 & $1.18 \times 10^{-2}$ \\
\hline & 245608_at & At4g14350 & Protein kinase family protein & -1.54 & $1.27 \times 10^{-6}$ \\
\hline & 253263_at & At4g34000 & ABA-responsive elements-binding factor 3 (ABF3) & 3.35 & $2.09 \times 10^{-11}$ \\
\hline \multirow[t]{4}{*}{48} & 253399_at & At4g32850 & Nuclear poly(A) polymerase (nPAP) & 0.7 & $2.22 \times 10^{-2}$ \\
\hline & 256683_at & At3g52220 & Expressed protein & -0.47 & $4.43 \times 10^{-2}$ \\
\hline & 256940_at & At3g30720 & Expressed protein & 1.68 & $3.25 \times 10^{-2}$ \\
\hline & 262719_at & At1g43590 & Transposable element gene & 2.02 & $3.12 \times 10^{-6}$ \\
\hline \multirow[t]{4}{*}{57} & 253263_at & At4g34000 & ABA-responsive elements-binding factor 3 (ABF3) & 4.44 & $2.58 \times 10^{-14}$ \\
\hline & 244992_s_at & AtCg00900/AtCg01240 & Ribosomal protein s7 (rps7)* & -1.68 & $4.80 \times 10^{-3}$ \\
\hline & 244996_at & AtCg00160 & Ribosomal protein s2 (rps2)* & -1.28 & $9.05 \times 10^{-3}$ \\
\hline & 245018_at & AtCg00520 & Protein required for photosystem I assembly and stability $(y c f 4)^{*}$ & -1.22 & $9.22 \times 10^{-3}$ \\
\hline \multirow[t]{5}{*}{59} & 245019_at & AtCg00530 & Hypothethical protein $(y c f 10 / c e m A)^{*}$ & -1.34 & $1.48 \times 10^{-2}$ \\
\hline & 245020_at & AtCg00540 & Cytochrome $f$ apoprotein $($ petA)* & -1.21 & $1.25 \times 10^{-2}$ \\
\hline & 253263_at & At4g34000 & ABA-responsive elements-binding factor 3 (ABF3) & 2.04 & $1.35 \times 10^{-6}$ \\
\hline & 261888_at & At1g80800 & Pseudogene, 40S ribosomal protein S12 (rps12B) & -0.92 & $2.31 \times 10^{-2}$ \\
\hline & 264683_at & At1g65580 & Inositol or phosphatidylinositol phosphatase/FRAGILE FIBRE 3 (FRA3) & -0.71 & $2.34 \times 10^{-2}$ \\
\hline
\end{tabular}

$M$ is $\log 2$ fold change and $P$ is FDR-adjusted $P$-value for Student's $t$-test.

* indicates genes encoded by the chloroplast genome. 
genes were differentially expressed in control plants exposed to drought stress compared to unstressed control plants and 797 genes were differentially expressed in 35S:ABF3 plants exposed to drought stress compared to unstressed 35S:ABF3 plants.

In order to compare the profile of genes expressed in the two plant lines in response to drought stress, functional categorizations of the differentially expressed genes were obtained from The Arabidopsis Information Resource (TAIR; http://www.arabidopsis.org) and compared. Although the profile of genes differentially expressed in control and 35S:ABF3 plants show some differences, overall they show a similar pattern of distribution among the different functional classes (Additional file 2). The greatest difference between 35S:ABF3 and control plant lines in the percentage of genes belonging to a particular functional category was $1.3 \%$ for the 'other metabolic processes' category at the $2 \mathrm{~h}$ time point. This suggests that the overall functional response of $35 \mathrm{~S}: \mathrm{ABF} 3$ and control plant lines at the gene expression level was similar.

In total, 1234 genes were differentially expressed in at least one plant line during at least one time point. The overlap in genes expressed in the two plant lines at the two different time points is depicted with a four-way Venn diagram in Figure 3. These genes can be subdivided into three categories. There are 564 genes that were differentially expressed in both control and 35S: ABF3 lines at the same time points suggesting that they were commonly regulated in both lines. There are 407 genes that are regulated differently in control and 35S: ABF3 plant lines that show an enhanced response in the 35S:ABF3 line. Finally, there are 263 genes that are regulated differently in control and 35S:ABF3 plant lines that show an attenuated response in the 35S:ABF3 line. In the latter two categories, these genes are either uniquely differentially expressed in one line or the other, or they are differentially expressed in one line at one time point but not in the other line at that time point. Since only two time points were examined, it is difficult to determine if the observed differences in the two lines are due to differences in the timing of gene expression or in the magnitude of gene expression or some combination of both of these factors. Additional file 3 contains a list of all of the differentially expressed genes found in each of the three categories.

In order to confirm the microarray results, RT-PCR was performed on 32 genes from the enhanced and attenuated categories. Twenty-nine of the examined genes exhibited expression patterns that were consistent with the microarray results, confirming the reliability of the microarray data (Figure 4). Amplification of one gene, At2g22760, produced two bands, one corresponding to the expected size of $526 \mathrm{bp}$. The primers for this gene were designed around an intron and the size of the second band is similar to the $664 \mathrm{bp}$ that would be expected if the intron were not spliced, suggesting that this band may represent a splice variant.

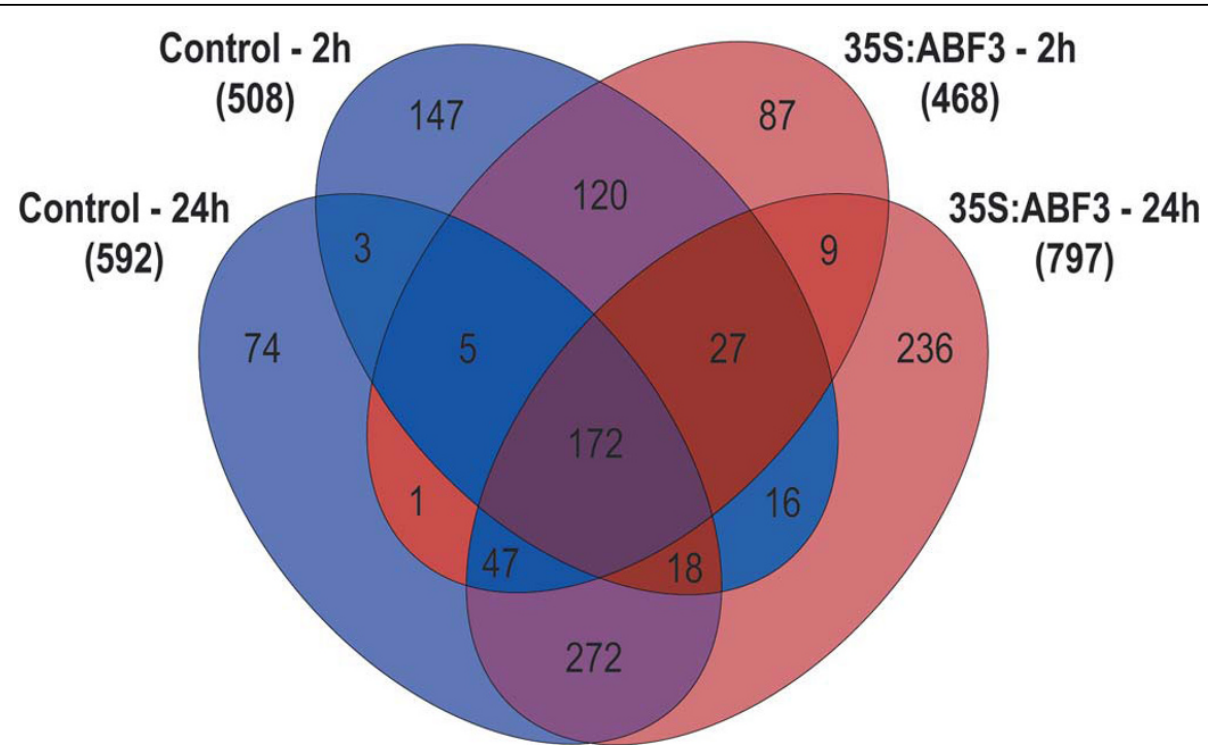

Figure 3 Overlap of differentially expressed genes in 35S:ABF3 and control plants in response to drought. Four-way Venn diagram showing the overlap of differentially expressed genes in 35S:ABF3 and control plant lines in response to drought at $2 \mathrm{~h}$ and $24 \mathrm{~h}$. Regions corresponding to genes that are regulated commonly in both plant lines are shaded in purple. Regions corresponding to genes that show enhanced regulation in 35S:ABF3 plants are shaded in red and those that show attenuated regulation in 35S:ABF3 plants are shaded in blue. 


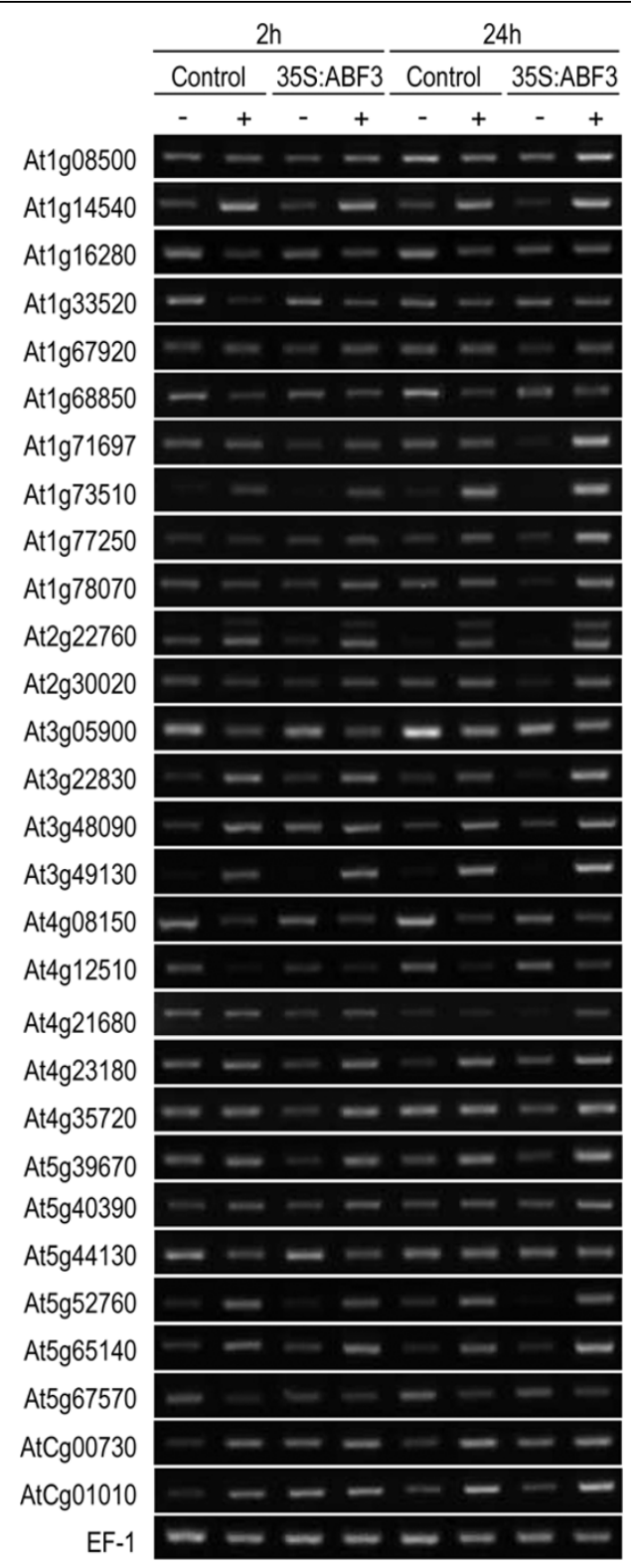

Figure 4 RT-PCR confirmation of microarray data. RT-PCR was performed using RNA from unstressed (-) or drought stressed (+) control and $35 \mathrm{~S}: \mathrm{ABF} 3$ plants at either the $2 \mathrm{~h}$ or $24 \mathrm{~h}$ time point. Genes showing either enhanced or attenuated regulation in $35 \mathrm{~S}: \mathrm{ABF} 3$ plants in response to drought were amplified using gene-specific primers. Elongation factor 1- $\alpha$ (EF-1) was amplified as an internal control.

\section{Genes commonly regulated in both 35S:ABF3 and control} lines

There are 564 genes that are commonly regulated in both 35S:ABF3 and control lines. Of these, 172 show differential expression in both lines at both time points, 120 are only differentially expressed at $2 \mathrm{~h}$ and 272 are only differentially expressed at $24 \mathrm{~h}$.

A number of genes in this category are known to act in pathways that are upstream or independent of ABF3, which is consistent with their common pattern of regulation in both 35S:ABF3 and control plant lines
(Table 3). Since ABF3 is an ABA-dependent transcription factor, ABA biosynthesis should occur upstream of ABF3 activity. Consistent with this, AtNCED3 (At3g14440), which encodes a 9-cis-epoxycarotenoid dioxygenase enzyme involved in ABA biosynthesis, was upregulated in both lines at both time points in response to drought treatment as was the gene CYP707A3 (At5g45340), which encodes a cytochrome P450 monooxygenase involved in ABA catabolism.

Several transcription factors have been identified that mediate drought response pathways independent from 
Table 3 Genes commonly regulated in 35S:ABF3 and control plant lines that act in pathways upstream or independent from ABF3

\begin{tabular}{|c|c|c|c|c|c|c|}
\hline \multirow[b]{2}{*}{ Probe Set ID } & \multirow[b]{2}{*}{ AGI } & \multirow[b]{2}{*}{ Annotation } & \multicolumn{2}{|c|}{$2 \mathrm{~h}$} & \multicolumn{2}{|r|}{$24 \mathrm{~h}$} \\
\hline & & & Control & $35 \mathrm{~S}: \mathrm{ABF} 3$ & Control & 35S:ABF3 \\
\hline \multicolumn{7}{|c|}{ ABA Metabolism } \\
\hline 257280_at & At3g14440 & AtNCED3 & 3.653 & 4.141 & 2.385 & 2.834 \\
\hline 248964_at & At5g45340 & CYP707A3 & 2.007 & 2.192 & 3.177 & 3.399 \\
\hline \multicolumn{7}{|c|}{ DREB Transcription Factors } \\
\hline 250781_at & At5g05410 & DREB2A & 3.945 & 4.672 & 4.755 & 4.602 \\
\hline 256430_at & At3g11020 & DREB2B & 1.376 & 1.562 & 1.94 & 1.987 \\
\hline \multicolumn{7}{|c|}{ NAC Transcription Factors } \\
\hline 260203_at & At1g52890 & ANAC019 & 3.945 & 4.409 & 4.264 & 4.608 \\
\hline 258395_at & At3g15500 & ANAC055/AtNAC3 & 3.088 & 3.258 & 2.297 & 2.557 \\
\hline 253872_at & At4g27410 & ANAC072/RD26 & 2.602 & 3.183 & 2.567 & 2.594 \\
\hline
\end{tabular}

Values are log2 fold changes.

Table 4 Examples of typical drought-responsive genes regulated similarly in 35S:ABF3 and control plant lines

\begin{tabular}{|c|c|c|c|c|c|c|}
\hline \multirow[b]{2}{*}{ Probe Set ID } & \multirow[b]{2}{*}{ AGI } & \multirow[b]{2}{*}{ Annotation } & \multicolumn{2}{|c|}{$2 \mathrm{~h}$} & \multicolumn{2}{|c|}{$24 \mathrm{~h}$} \\
\hline & & & Control & 35S:ABF3 & Control & 35S:ABF3 \\
\hline \multicolumn{7}{|c|}{ Osmolyte metabolism } \\
\hline 251505_at & At3g59050 & Polyamine oxidase 3 & & & 1.365 & 1.409 \\
\hline 266072_at & At2g18700 & Trehalose-phosphatase/synthase 11 (AtTPS11) & 1.173 & 1.290 & & \\
\hline 254321_at & At4g22590 & Trehalose-6-phosphate phosphatase, putative & & & 2.262 & 2.652 \\
\hline 250467_at & At5g10100 & Trehalose-6-phosphate phosphatase, putative & & & 1.716 & 1.560 \\
\hline \multicolumn{7}{|c|}{ LEA class proteins } \\
\hline 250648_at & At5g06760 & LEA group 1 domain-containing protein & 2.281 & 2.823 & 2.537 & 3.013 \\
\hline 262128_at & At1g52690 & LEA protein, putative & 3.087 & 3.667 & & \\
\hline 267261_at & At2g23120 & Expressed protein & & & 1.651 & 1.688 \\
\hline 266392_at & At2g41280 & LEA protein M10 & & & 2.258 & 2.473 \\
\hline 252988_at & At4g38410 & Dehydrin, putative & & & 2.692 & 2.516 \\
\hline \multicolumn{7}{|l|}{ Kinases } \\
\hline 252592_at & At3g45640 & MAPK, putative (MPK3) & 1.681 & 1.772 & 1.259 & 1.651 \\
\hline 258682_at & At3g08720 & Serine/threonine protein kinase (PK19) & & & 1.160 & 1.383 \\
\hline 250673_at & At5g07070 & CBL-interacting protein kinase 2 (CIPK2) & 1.408 & 1.261 & 1.551 & 1.827 \\
\hline 254996_at & At4g10390 & Protein kinase family protein & & & 2.177 & 1.763 \\
\hline 249361_at & At5g40540 & Protein kinase, putative & & & 2.768 & 3.066 \\
\hline 248821_at & At5g47070 & Protein kinase, putative & & & 1.547 & 1.487 \\
\hline \multicolumn{7}{|l|}{ Phosphatases } \\
\hline 247957_at & At5g57050 & Abscisic acid-insensitive 2 (ABI2) & 1.617 & 2.228 & & \\
\hline 247723_at & At5g59220 & Protein phosphatase $2 \mathrm{C}$, putative & 1.675 & 2.115 & & \\
\hline 251259_at & At3g62260 & Protein phosphatase $2 \mathrm{C}$, putative & 2.533 & 2.710 & 2.902 & 2.440 \\
\hline 253323_at & At4g33920 & Protein phosphatase $2 \mathrm{C}$ family protein & 1.385 & 1.615 & 1.767 & 1.779 \\
\hline \multicolumn{7}{|c|}{ Transcription factors } \\
\hline 250582_at & At5g07580 & Ethylene-responsive element-binding family protein & -1.878 & -1.668 & & \\
\hline 265452_at & At2g46510 & Basic helix-loop-helix family protein & & & 1.918 & 2.460 \\
\hline 247509_at & At5g62020 & Heat shock transcription factor B2A (AtHSFB2A) & 1.133 & 1.135 & 1.594 & 1.900 \\
\hline 251272_at & At3g61890 & Homeobox-leucine zipper protein 12 (HB-12) & 2.421 & 2.536 & 1.914 & 2.554 \\
\hline 260237_at & At1g74430 & Myb family transcription factor (MYB95) & & & 1.931 & 1.942 \\
\hline 255753_at & At1g18570 & Myb family transcription factor (MYB51) & 2.127 & 1.409 & & \\
\hline 254652_at & At4g18170 & WRKY family transcription factor (WRKY28) & 3.564 & 2.709 & & \\
\hline 261648_at & At1g27730 & Zinc finger ( $\mathrm{C} 2 \mathrm{H} 2$ type) family protein (ZAT10) & 3.665 & 3.663 & 4.978 & 5.127 \\
\hline
\end{tabular}

Values are $\log 2$ fold changes. 
Table 5 Genes showing an enhanced response in 35S:ABF3 plants that may contribute to drought-tolerance

\begin{tabular}{|c|c|c|c|c|c|c|}
\hline \multirow[b]{2}{*}{$\begin{array}{l}\text { Probe Set } \\
\text { ID }\end{array}$} & \multirow[b]{2}{*}{ AGI } & \multirow[b]{2}{*}{ Annotation } & \multicolumn{2}{|c|}{$2 \mathrm{~h}$} & \multicolumn{2}{|c|}{$24 \mathrm{~h}$} \\
\hline & & & Control & $\begin{array}{l}\text { 35S: } \\
\text { ABF3 }\end{array}$ & Control & $\begin{array}{l}\text { 35S: } \\
\text { ABF3 }\end{array}$ \\
\hline \multicolumn{7}{|c|}{ Osmolyte metabolism } \\
\hline 248404_at & At5g51460 & Trehalose-6-phosphate phosphatase (TPPA) & & & & 1.402 \\
\hline 247228_at & At5g65140 & Trehalose-6-phosphate phosphatase, putative & 1.479 & 1.587 & & 2.224 \\
\hline 263452_at & At2g22190 & Trehalose-6-phosphate phosphatase, putative & & 1.250 & & \\
\hline 254806_at & At4g12430 & Trehalose-6-phosphate phosphatase, putative & & 1.146 & & \\
\hline 252983_at & At4g37980 & Mannitol dehydrogenase, putative/elicitor activated gene 3 (ELI3-1) & & & & -1.061 \\
\hline 251729_at & At3g56310 & Alpha-galactosidase, putative & & & & -1.160 \\
\hline \multicolumn{7}{|c|}{ Detoxification } \\
\hline 266299_at & At2g29450 & Glutathione S-transferase (class tau) 5 (AtGSTU5) & & & & 2.268 \\
\hline 258665_at & At3g08710 & Thioredoxin H-type 9 (ATH9) & & & & 1.188 \\
\hline \multicolumn{7}{|c|}{ LEA class proteins } \\
\hline 259516_at & At1g20450 & Dehydrin/early response to dehydration 10 (ERD10) & & & & 1.584 \\
\hline 252102_at & At3g50970 & dehydrin/XERO2/low-temperature-induced protein & & & & 2.800 \\
\hline 265211_at & At2g36640 & LEAprotein/embryonic cell protein 63 (AtECP63) & & 1.652 & & \\
\hline \multicolumn{7}{|l|}{ Transporters } \\
\hline 249063_at & At5g44110 & $A B C$ transporter family protein & & 1.017 & 1.540 & 1.826 \\
\hline 263918_at & At2g36590 & Proline transporter 3 (ProT3) & & 1.907 & & 1.845 \\
\hline 245868_at & At1g58030 & Cationic amino acid transporter 2 (CAT2) & & & & -1.406 \\
\hline 260543_at & At2g43330 & Inositol transporter 1 (AtINT1) & & 1.118 & & 0.160 \\
\hline 262756_at & At1g16370 & $\begin{array}{l}\text { Organic cation/carnitine transporter } 6 \text { (AtOCT6)/carbohydrate } \\
\text { transmembrane transporte }\end{array}$ & & & & 1.992 \\
\hline 245499_at & At4g16480 & Inositol transporter 4 (AtINT4) & & & & -1.031 \\
\hline 254291_at & At4g23010 & UDP-galactose transporter 2 (AtUTR2) & & & & 1.081 \\
\hline 267423_at & At2g35060 & K+ uptake permease 11 (KUP11)/potassium transporter family protein & & & & -1.202 \\
\hline 249298_at & At5g41330 & Potassium channel tetramerisation domain-containing protein & & & & -1.157 \\
\hline 256402_at & At3g06130 & Heavy-metal-associated domain-containing protein & & & & -1.363 \\
\hline 247128_at & At5g66110 & Heavy-metal-associated domain-containing protein & & & & -1.716 \\
\hline 261143_at & At1g19770 & Purine permease 14 (AtPUP14); purine transmembrane transporter & 1.222 & 1.571 & & 1.569 \\
\hline 262649_at & At1g14040 & EXS family protein/ERD1/XPR1/SYG1 family protein & & & & 1.103 \\
\hline 250151_at & At5g14570 & High affinity nitrate transporter 2.7 (AtNRT2.7) & & & & -1.317 \\
\hline 251916_at & At3g53960 & Proton-dependent oligopeptide transport (POT) family protein & & 1.278 & & 1.548 \\
\hline 252589_s_at & $\begin{array}{l}\text { At3g45650/ } \\
\text { At3g45660 }\end{array}$ & Proton-dependent oligopeptide transport (POT) family protein & & 1.164 & & 0.740 \\
\hline 254396_at & At4g21680 & Proton-dependent oligopeptide transport (POT) family protein & & & & 2.426 \\
\hline
\end{tabular}

the ABF3 pathway. Members of the DREB family of transcription factors function in abiotic stress signalling and members of the DREB2 subfamily are known to function in ABA-independent drought stress signalling [59]. DREB2A (At5g05410) and DREB2B (At3g11020), as expected, were upregulated in response to drought in both plant lines at both time points (Table 3). In addition, DREB1B/CBF1 (At4g25490) and DREB1C/CBF2 (At4g25470) were also upregulated in both lines at both time points and DDF1 (At1g12610) was upregulated at 24 h (Additional file 3). While the DREB1 subfamily is primarily associated with cold stress signalling [59], there is some evidence that members also function in dehydration stress $[8,38,60,61]$.
Three NAC transcription factors also function in a drought signalling pathway that is independent from ABF3 [42]. ANAC19 (At1g52890), ANAC055/ATNAC3 (At3g15500), and ANAC072/RD26 (At4g27410) are all expressed at both time points in both plant lines (Table 3 ). Another six members of the NAC family of transcription factors (At1g01720, At1g69490, At1g77450, At2g02450, At3g49530, and At5g63790) are also commonly regulated in both $35 \mathrm{~S}: \mathrm{ABF} 3$ and control plant lines (Additonal file 3).

Also in this category are many genes that are known to be regulated in response to drought stress. This includes genes involved in the biosynthesis of osmolytes, late embryogenesis abundant proteins (LEA), 
Table 6 Potential targets of ABF3

\begin{tabular}{|c|c|c|c|c|c|c|c|}
\hline \multirow{2}{*}{\multicolumn{2}{|c|}{ Probe Set ID }} & \multirow[b]{2}{*}{ Annotation } & \multirow[b]{2}{*}{ ABREs $^{1}$} & \multicolumn{2}{|c|}{$2 \mathrm{~h}$} & \multicolumn{2}{|c|}{$24 \mathrm{~h}$} \\
\hline & & & & Control & 35S:ABF3 & Control & 35S:ABF3 \\
\hline \multicolumn{8}{|c|}{ Osmolyte biosynthesis } \\
\hline 254806_at & At4g12430 & Trehalose-6-phosphate phosphatase, putative & 1 & & 1.146 & & \\
\hline \multicolumn{8}{|c|}{ Protein degradation } \\
\hline 262164_at & At1g78070 & WD-40 repeat family protein & 1 & & 2.072 & 2.312 & 2.647 \\
\hline \multicolumn{8}{|l|}{ Signalling } \\
\hline 261506_at & At1g71697 & Choline kinase, putative (AtCK1) & 1 & 1.519 & 1.181 & & 2.909 \\
\hline \multicolumn{8}{|l|}{ Transcription } \\
\hline 253259_at & At4g34410 & AP2 domain-containing transcription factor, putative & 2 & & 3.865 & 5.126 & 4.940 \\
\hline 259432_at & At1g01520 & Myb family transcription factor & 3 & 2.367 & 2.762 & & 3.247 \\
\hline 262098_at & At1g56170 & Nuclear factor Y, subunit C2 (NF-YC2) & 3 & & 1.944 & & \\
\hline 261892_at & At1g80840 & WRKY family transcription factor (WRKY40) & 2 & & 3.762 & 4.724 & 5.138 \\
\hline \multicolumn{8}{|l|}{ Transporters } \\
\hline 260543_at & At2g43330 & Inositol transporter 1 (AtINT1) & 1 & & 1.118 & & \\
\hline 251916_at & At3g53960 & Proton-dependent oligopeptide transport (POT) family protein & 2 & & 1.278 & & 1.548 \\
\hline \multicolumn{8}{|l|}{ Other } \\
\hline 251904_at & At3g54130 & Josephin family protein & 1 & & 1.043 & & 1.311 \\
\hline 265634_at & At2g25530 & AFG1-like ATPase family protein & 2 & & & & 1.399 \\
\hline 252557_at & At3g45960 & Expansin family protein (EXPL3) & 1 & & 1.467 & & 1.850 \\
\hline 253217_at & At4g34970 & Actin-depolymerizing factor, putative & 1 & & -1.417 & -2.589 & -2.791 \\
\hline 262126_at & At1g59620 & Disease resistance protein (CC-NBS class), putative & 2 & & 1.235 & & \\
\hline 247246_at & At5g64620 & Invertase/pectin methylesterase inhibitor family protein & 1 & & -1.125 & & \\
\hline 246495_at & At5g16200 & $50 S$ ribosomal protein-related & 1 & & 1.193 & 2.080 & 2.162 \\
\hline \multicolumn{8}{|l|}{ Unknown } \\
\hline 261193_at & At1g32920 & Expressed protein & 1 & & 1.541 & 1.793 & 2.457 \\
\hline 260227_at & At1g74450 & Expressed protein & 3 & & 1.755 & 1.840 & 2.170 \\
\hline 253155_at & At4g35720 & Expressed protein & 3 & & 2.671 & & 2.010 \\
\hline 261065_at & At1g07500 & Expressed protein & 2 & & 1.201 & & \\
\hline 256069_at & At1g13740 & Expressed protein & 5 & & 2.090 & & \\
\hline 260367_at & At1g69760 & Expressed protein & 1 & & -1.278 & & \\
\hline 260005_at & At1g67920 & Expressed protein & 1 & 2.251 & 2.051 & & 2.825 \\
\hline 254356_at & At4g22190 & Expressed protein & 2 & -1.118 & -1.031 & & -1.423 \\
\hline
\end{tabular}

Values are $\log 2$ fold changes.

${ }^{1}$ Number of ABA-responsive elements (ABREs) in the promoter region as determined by Gómez-Porras et al. [62]

kinases, phosphatases, and transcription factors, as well as several different types of transporters (Table 4). This further suggests that some drought signalling pathways are unaffected by $A B F 3$ overexpression

\section{Genes with an enhanced response in 35S:ABF3 plants}

There are 407 genes that demonstrated an enhanced response in 35S:ABF3 plants. Of these, 332 are uniquely regulated in 35S:ABF3 plants with 9 differentially expressed at both time points, 87 differentially expressed only at $2 \mathrm{~h}$ and 236 differentially expressed at $24 \mathrm{~h}$. In addition, 47 genes are differentially expressed in the control line at only $24 \mathrm{~h}$ and 27 genes are differentially expressed in the control line at only $2 \mathrm{~h}$ while in the 35S:ABF3 line they are differentially expressed at both time points. Finally, there is a transposable element gene that is differentially expressed in 35S:ABF3 plants at $2 \mathrm{~h}$ but is only differentially expressed in control plants at $24 \mathrm{~h}$.

A number of genes in this category could contribute to the enhanced drought tolerance of 35S:ABF3 plants (Table 5). Several genes involved in the biosynthesis of the osmolyte trehalose show enhanced upregulation while two genes involved in osmolyte catabolism show enhanced downregulation. Two genes involved in detoxification show enhanced upregulation in 35S:ABF3 as do three LEA proteins. A number of transporters also show enhanced regulation in 35S:ABF3 lines.

Direct targets of ABF3 are likely found in this category of genes. In addition to showing enhanced regulation in 35S:ABF3 plants, it is expected that these genes possess at least one ABRE in their promoter. In addition, these 
Table 7 Genes showing enhanced regulation in 35S:ABF3 with predicted function in RNA processing

\begin{tabular}{|c|c|c|c|c|c|c|}
\hline \multirow[b]{2}{*}{ Probe Set ID } & \multirow[b]{2}{*}{ AGI } & \multirow[b]{2}{*}{ Annotation } & \multicolumn{2}{|c|}{$2 \mathrm{~h}$} & \multicolumn{2}{|c|}{$24 \mathrm{~h}$} \\
\hline & & & Control & 35S:ABF3 & Control & 35S:ABF3 \\
\hline \multicolumn{7}{|c|}{ Splicing and splicing-related } \\
\hline 262110_at & At1g02840 & Pre-mRNA splicing factor SF2/SR1 protein & & & & -1.731 \\
\hline 263035_at & At1g23860 & Splicing factor RSZp21 (RSZP21) & & & & -1.272 \\
\hline 262295_at & At1g27650 & U2 snRNP auxiliary factor small subunit, putative (AtU2AF35A) & & & & -1.129 \\
\hline 262931_at & At1g65700 & Small nuclear ribonucleoprotein, putative & & & & 1.386 \\
\hline 267102_at & At2g41500 & LACHESIS (LIS)/related to yeast splicing factor PRP4 & & & & -1.260 \\
\hline 252182_at & At3g50670 & U1 small nuclear ribonucleoprotein 70 (U1-70k) & & & & -1.525 \\
\hline 251798_at & At3g55460 & SC35-like splicing factor, 30 kD (SCL30) & & & & -1.048 \\
\hline 253668_at & At4g30220 & Small nuclear ribonucleoprotein $\mathrm{F}$ (RUXF), putative & & & & 1.057 \\
\hline 249870_at & At5g23080 & SWAP domain-containing protein/TOUGH (TGH) & & & & -1.476 \\
\hline 246924_at & At5g25060 & RNA recognition motif (RRM)-containing protein & & & & -1.043 \\
\hline 248369_at & At5g52040 & Arginine/serine-rich splicing factor RSP41 (RSP41) & & & & -1.009 \\
\hline \multicolumn{7}{|c|}{ Nucleocytoplasmic transport } \\
\hline 257817_at & At3g25150 & Nuclear transport factor 2 (NTF2) family protein & & & & -1.316 \\
\hline \multicolumn{7}{|l|}{ Deadenylation } \\
\hline 252679_at & At3g44260 & CCR4-NOT transcription complex protein, putative & & 2.108 & 3.479 & 3.354 \\
\hline \multicolumn{7}{|l|}{ Other } \\
\hline 262804_at & At1g20880 & RNA recognition motif (RRM)-containing protein & & & & -1.335 \\
\hline 261988_at & At1g33680 & KH domain-containing protein & & & & -1.123 \\
\hline 254355_at & At4g22380 & Ribosomal protein L7Ae/L30e/S12e/Gadd45 family protein & & & & 1.617 \\
\hline 246088_at & At5g20600 & Expressed protein & & & & -1.145 \\
\hline 247004_at & At5g67570 & Pentatricopeptide (PPR) repeat-containing protein & & & & -1.349 \\
\hline
\end{tabular}

Values are log2 fold changes.

genes are likely to be significantly differentially expressed at the $2 \mathrm{~h}$ time point. Amongst those genes showing an enhanced response in 35S:ABF3 plants that are significantly differentially expressed at the $2 \mathrm{~h}$ time point, 24 contain at least one ABRE according to the in silico analysis performed by Gómez-Porras et al [62] and are therefore identified as putative ABF3 targets (Table 6). Included in this group are four transcription factors, one choline kinase, one trehalose biosynthetic enzyme, one gene involved in ubiquitin-mediated protein degradation, and two transporters as well as seven other genes with undefined roles in the drought response and eight unknown genes.

Amongst those genes that show an enhanced response in 35S:ABF3 plants, there is an enrichment of genes that function in RNA processing pathways (Table 7). There are 8 genes involved in RNA processing that are similarly regulated in both control and 35S:ABF3 plants lines, 8 genes that show an attenuated response in 35S: ABF3, while 18 genes show an enhanced response. Many of these appear to be uniquely downregulated in 35S:ABF3 plants at $24 \mathrm{~h}$. Eleven of these are predicted to function in RNA splicing or to be associated with the RNA splicing machinery while the others have roles in nucleocytoplasmic transport, deadenylation, or the function is not specifically known. Several genes that function in RNA processing have been found to function in $\mathrm{ABA}$ and abiotic stress signalling pathways [63-66]. This suggests that the differential regulation of RNA processing genes in 35S:ABF3 lines could contribute to its enhanced drought tolerance.

Interestingly, a member of the DREB family of transcription factors is also included in this group. DREB1D/CBF4 (At5g51990) was upregulated in 35S: ABF3 plants at both time points but was only upregulated in control plants at $24 \mathrm{~h}$. DREB1D/CBF4 functions in both cold and drought stress signalling and, unlike the DREB2 subfamily, its expression is induced by ABA [38]. This suggests that CBF4 may act downstream of ABF3 in the ABA-dependent drought signalling pathway.

\section{Genes with an attenuated response in 35S:ABF3 plants}

There are 263 genes that show an attenuated response in 35S:ABF3 plants. Of these, 224 are uniquely regulated in control lines with 3 differentially expressed at both time points, 147 differentially expressed only at $2 \mathrm{~h}$ and 74 differentially expressed only at $24 \mathrm{~h}$. In addition, 18 genes are differentially expressed in the 35S:ABF3 line at only $24 \mathrm{~h}$ and 5 genes are differentially expressed in the 35S:ABF3 line at only $2 \mathrm{~h}$ while in the control line they are differentially expressed at both time points. 
Table 8 Genes encoded by the chloroplast and mitochondrial genomes showing attenuated regulation in 35S:ABF3 plants

\begin{tabular}{|c|c|c|c|c|c|c|}
\hline \multicolumn{2}{|c|}{ Probe Set } & \multirow[b]{2}{*}{ Annotation } & \multicolumn{2}{|c|}{$2 \mathrm{~h}$} & \multicolumn{2}{|c|}{$24 \mathrm{~h}$} \\
\hline ID & AGI & & Control & 35S:ABF3 & Control & 35S:ABF3 \\
\hline \multicolumn{7}{|c|}{ Electron transport activity } \\
\hline 266045_s_at & $\begin{array}{l}\text { At2g07727/ } \\
\text { AtMg00220 }\end{array}$ & $\begin{array}{l}\text { Cytochrome b (MTCYB) (COB) (CYTB)/apocytochrome b } \\
\text { (cob) }^{\dagger}\end{array}$ & 2.060 & & & \\
\hline 244903_at & AtMg00660 & Hypothetical protein (orf149) ${ }^{+}$ & 1.645 & & & \\
\hline 244977_at & AtCg00730 & Subunit IV of cytochrome b6/f complex (petD)* & 2.993 & & 3.118 & \\
\hline \multicolumn{7}{|c|}{ NADH dehydrogenases } \\
\hline 244943_at & AtMg00070 & $\mathrm{NADH}$ dehydrogenase subunit $9(\operatorname{nad} 9)^{\dagger}$ & 1.338 & & & 1.416 \\
\hline 257337_at & AtMg00060 & $\begin{array}{l}\text { NADH dehydrogenase subunit } 5 \text { (nad5) (Transplice part } 3 \\
\text { of 3) }\end{array}$ & 1.413 & & & \\
\hline 244933_at & AtCg01070 & NADH dehydrogenase ND4L (ndhE)* & 1.733 & & & \\
\hline 244994_at & AtCg01010 & Chloroplast encoded NADH dehydrogenase unit (ndhF)* & 3.240 & & & \\
\hline 244934_at & AtCg01080 & NADH dehydrogenase ND6 (ndhG)* & 2.465 & & & \\
\hline 244991_s_at & $\begin{array}{l}\text { AtCg00890/ } \\
\text { AtCg01250 }\end{array}$ & NADH dehydrogenase ND2 (ndhB)* & 3.528 & & & \\
\hline \multicolumn{7}{|c|}{ RNA processing } \\
\hline 244999_at & AtCg00190 & RNA polymerase subunit beta $(r p o B)^{*}$ & 2.792 & & & \\
\hline \multicolumn{7}{|l|}{ Translation } \\
\hline 245005_at & AtCg00330 & Chloroplast ribosomal protein s14 (rps14)* & 3.796 & & 3.644 & \\
\hline 244970_at & AtCg00660 & Ribosomal protein L20 (rpl20)* & 2.379 & & & \\
\hline 244939_at & AtCg00065 & Ribosomal protein s12 (Transplice part 1 of 2) $(\operatorname{rps} 12.1)^{*}$ & 1.653 & & & \\
\hline \multicolumn{7}{|c|}{ ATPase subunits } \\
\hline 244995_at & AtCg00150 & Subunit of ATPase complex CF0 (atpl)* & 2.049 & & & \\
\hline 266012_s_at & $\begin{array}{l}\text { AtMg00410/ } \\
\text { AtMg01170/ } \\
\text { At2g07699/ } \\
\text { At2g07741 }\end{array}$ & ATPase subunit $\sigma^{\dagger}$ & 1.924 & & & \\
\hline \multicolumn{7}{|l|}{ Other } \\
\hline 257319_at & AtMg01100 & Hypothetical protein $\left(\right.$ orf105a) ${ }^{\dagger}$ & 1.181 & & & \\
\hline 244989_s_at & $\begin{array}{l}\text { AtCg00860/ } \\
\text { AtCg01280 }\end{array}$ & Expressed protein $(y c f 2)^{*}$ & 3.493 & & & \\
\hline 245008_at & AtCg00360 & $\begin{array}{l}\text { Protein required for photosystem I assembly and stability } \\
\left(y(f 3)^{*}\right.\end{array}$ & 2.371 & & & \\
\hline 244990_s_at & $\begin{array}{l}\text { AtCg00870/ } \\
\text { AtCg01270 }\end{array}$ & Hypothetical protein (ycf15/orf77)* & 3.607 & & & 2.841 \\
\hline 245016_at & AtCg00500 & $\begin{array}{l}\text { Acetyl-CoA carboxylase carboxyl transferase subunit beta } \\
(\text { accD)* }\end{array}$ & 1.452 & & & \\
\hline 266014_s_at & $\begin{array}{l}\text { At2g07722/ } \\
\text { AtMg00170/ } \\
\text { AtMg00620 }\end{array}$ & Hypothetical protein $^{\dagger}$ & 1.987 & & & \\
\hline
\end{tabular}

Values are $\log 2$ fold changes.

* indicates gene is encoded by the chloroplast genome.

${ }^{+}$indicates gene is encoded by the mitochondrial genome.

Finally, there are 16 genes that are differentially expressed in the control line at $2 \mathrm{~h}$ and in the 35S:ABF3 line at $24 \mathrm{~h}$.

Surprisingly, a number of genes that typically function in conferring drought tolerance show an attenuated response in 35S:ABF3 plants. This includes several peroxidases and glutaredoxins involved in detoxification, two genes involved in biosynthesis of the osmolytes raffinose and spermine, a heat shock protein, and a number of transporters (Additional file 3). Similarly a number of transcription factors and other signalling components also show an attenuated response in 35S: ABF3 plants. The reason for the attenuated response of these genes in 35S:ABF3 plants is not clear but might reflect differences in the physiological state of 35S:ABF3 plants due to their enhanced drought resistance.

A number of genes encoded by the chloroplast and mitochondrial genomes show an attenuated response in 
Table 9 Transposable element genes showing attenuated regulation in 35S:ABF3 plants

\begin{tabular}{|c|c|c|c|c|c|c|}
\hline \multirow[b]{2}{*}{ Probe Set ID } & \multirow[b]{2}{*}{ AGI } & \multirow[b]{2}{*}{ Annotation } & \multicolumn{2}{|c|}{$2 \mathrm{~h}$} & \multicolumn{2}{|c|}{$24 \mathrm{~h}$} \\
\hline & & & Control & 35S:ABF3 & Control & 35S:ABF3 \\
\hline \multicolumn{7}{|c|}{ Transposable element genes } \\
\hline 265709_at & At2g03540 & Transposable element gene & & & 1.305 & \\
\hline 263769_at & At2g06390 & Transposable element gene & 1.511 & & & \\
\hline 257777_x_at & At3g29210 & Transposable element gene & 1.117 & & & \\
\hline 257345_s_at & At3g33066 & Transposable element gene; gypsy-like retrotransposon family (Athila) & 2.379 & & & \\
\hline
\end{tabular}

Values are log2 fold changes.

35S:ABF3 plants (Table 8). Some of these genes encode proteins with electron transport activity or NADH dehydrogenase activity while others are predicted to function in transcription and translation processes. Most of these genes are upregulated in control lines only at the $2 \mathrm{~h}$ time point. The reason for the exclusive upregulation of these genes in the control line is not clear. The chloroplast NADH dehydrogenase (NDH) complex is predicted to be involved in cyclic electron transport around photosystem I, thereby dissipating energy and maintaining ATP supply under conditions of low $\mathrm{CO} 2$ availability following stomatal closure in response to stress [67]. The NDH complex may also be involved in detoxification in the chloroplast [68]. The upregulation of genes encoding NADH dehydrogenases may therefore reflect the greater sensitivity of the control plant line to stress. Rice plants overexpressing $A B F 3$ were able to maintain higher photochemical efficiency during drought stress [11]. This suggests that one aspect of the drought tolerance conferred by $A B F 3$ overexpression may be a minimization of the negative impact of drought stress on photosynthesis, which is reflected by differences in gene expression in the chloroplast. Similar effects may also occur in the mitochondria.

Four transposable element genes were also included in the attenuated response category (Table 9). Only one other transposable element gene was significantly differentially expressed and it was included in the enhanced regulation category. Many transposable elements are transcriptionally activated in response to stress conditions $[69,70]$. The attenuated regulation of transposable element genes in 35S:ABF3 plants might suggest that they are experiencing a lower level of stress that is insufficient to activate the transposable element genes, consistent with the drought tolerance of these plants.

Interestingly, $D R E B 1 A / C B F 3$ showed an attenuated response in 35S:ABF3 plants. DREB1A/CBF3 was upregulated in control plant lines at both time points but was only upregulated in 35S:ABF3 plants at $2 \mathrm{~h}$. This may again reflect differences in the tolerance of $35 \mathrm{~S}$ : ABF3 and control plants to the drought stress, with control plants requiring a stronger or longer activation of DREB1A/CBF3 expression in response to the increased stress.

\section{Discussion}

The Impact of Cre recombinase on the transcriptome is minimal

In order to eliminate position effects and focus on unintended pleiotropic effects of transcription factor overexpression, the Cre/lox recombination system was employed to create a series of control plant lines that contain the selectable marker at the site of transgene insertion but from which the $A B F 3$ transgene was excised. The use of the Cre/lox recombination system also allowed us to determine the impact of Cre recombinase on the transcriptome.

In tomato, petunia, tobacco, and to a lesser extent Arabidopsis, expression of Cre recombinase has resulted in abnormal phenotypes, including leaf chlorosis, stunted growth, and sterility $[49,50]$. Similarly, expression of Cre recombinase resulted in reduced proliferation and chromosomal abnormalities in cultured embryonic mouse cells [55,57], toxicity in dividing cells of Drosophila melanogaster [54], and chromosomal rearrangements in mouse spermatids leading to male sterility [56]. These abnormal effects of Cre recombinase are suspected to result from Cre-mediated recombination using cryptic lox sites that may be found in eukaryotic genomes. Cryptic lox sites that can be recognized by Cre recombinase have been identified in the genomes of yeast and humans as well as the chloroplast genome of tobacco $[52,53,71,72]$.

If cryptic lox sites exist in plant genomes, expression of Cre recombinase could induce deletions or inversions of genome segments or even chromosome translocations that could adversely impact the plant. These deletions or inversions may alter the expression of genes found within this segment, which would be detectable by microarray. In three independent plants lines in which Cre recombinase was employed to excise the $A B F 3$ transgene from the T-DNA insertion, only a small number of genes were found to be differentially expressed (Table 1). Of these, two genes were found to be differentially expressed in two out of the three control lines. The two genes are the chloroplast encoded rps7 (AtCg00900 and AtCg01240) and rps12.1 (AtCg00065) genes. In tobacco, one of the cryptic lox sites identified in the chloroplast genome is found just downstream of 
the start site of the rps $12.2 /$ rps 7 operon $[52,53]$. The altered expression of rps 7 might suggest that this cryptic lox site is conserved in Arabidopsis and may have undergone Cre-mediated recombination. Cre recombinase may therefore also be able to act on cryptic lox sites in the Arabidopsis chloroplast genome, resulting in a change in expression of the affected genes. It is, however, unclear how Cre recombinase is targeted to the chloroplast since it is only predicted to be targeted to the nucleus. Cre-mediated chloroplast genome deletions are not likely to be of great concern since chloroplast genomes containing deletions in essential genes are typically rapidly lost due to selection pressures [73-75], especially once the Cre recombinase has been removed. The impact of Cre recombinase on the nuclear transcriptome was negligible, which demonstrates that in Arabidopsis this technology does not produce unintended effects.

\section{The activity of $A B F 3$ is strictly controlled}

Microarray analysis of Arabidopsis plants overexpressing the transcription factor $A B F 3$ suggests that alterations to the transcriptome are minimal when position effects are eliminated as a source of variation. In the absence of stress, a small number of genes were differentially expressed in three 35S:ABF3 plant lines (Table 2), but no genes were differentially expressed in more than one independent line.

Members of the ABF/AREB family of transcription factors bind to ABREs found in the promoters of ABAresponsive genes [29,31]. If the genes identified by microarray analysis are actual downstream targets of ABF3, they would be expected to contain at least one ABRE. An in silico analysis of the Arabidopsis nuclear genome has identified 3829 genes containing one or more ABREs [62]. None of the nuclear genes identified by microarray analysis of 35S:ABF3 transgenic plants are predicted to contain an ABRE. Other members of the ABF/AREB subfamily of transcription factors localize to the nucleus [76,77], and it is likely that ABF3 similarly functions in the nucleus. Therefore, it is unlikely that the chloroplast genes identified by microarray analysis are functional targets of ABF3. This suggests that overexpression of $A B F 3$ alone is not sufficient to alter the transcriptome.

This result was unexpected as previous work identified a number of genes with altered expression in Arabidopsis [7] and rice [11] overexpressing $A B F 3$. Similarly, overexpression studies of many other transcription factors have revealed alterations in gene expression and this approach is typically used to identify the gene network controlled by that particular transcription factor $[39,42,58]$. The absence of differentially expressed genes in 35S:ABF3 transgenic plants suggests that an additional signal is required to activate ABF3 that is not present in unstressed plants.

There is accumulating evidence that members of the ABF/AREB family of transcription factors are regulated by phosphorylation. ABF2/AREB1 transactivation of a reporter gene in the presence of $A B A$ was inhibited by the addition of the protein kinase inhibitor staurosporine [31] and ABI5 is phosphorylated following ABA treatment [78]. Several studies have suggested a role for members of the SnRK2 family of protein kinases in the phosphorylation of $A B F / A R E B$ transcription factors [79-84]. ABF3 and ABF4/AREB2 interact with the calcium-dependent protein kinase AtCPK32 and evidence suggests that it phosphorylates a highly conserved serine residue in ABF4/AREB2 that is necessary for activity [85]. The protein kinases CPK4 and CPK11 are also likely to phosphorylate ABF1 and ABF4/AREB2 and their activity is enhanced by $A B A$ [86]. It is possible that in the absence of stress, ABF3 is not phosphorylated and therefore cannot activate gene expression.

Furthermore, other factors necessary for the activity of ABF3 may not be expressed in the absence of abiotic stress. Members of the ABF/AREB family have been shown to interact with diverse proteins that are predicted to modulate their transcriptional activity. ABF2/ AREB1 interacts with an arm-repeat protein that is predicted to positively regulate its activity [87]. ABI5, $\mathrm{ABF} 1, \mathrm{ABF} 3$, and ABF4/AREB2 can interact with the transcription factor ABI3 $[88,89]$. Furthermore, the $r d 29 a$ promoter contains both an ABRE as well as a dehydration-responsive elements that is bound by members of the DREB/CBF family of transcription factors and the two elements function interdependently to activate expression of $r d 29 a$ [90]. Members of the ABF/ AREB can also heterodimerize [91], suggesting that other members of this family may need to be expressed in order for ABF3 to be functional. Therefore, it is possible that other components of the stress response pathway are necessary in order for ABF3 to be active, preventing $\mathrm{ABF} 3$ from altering gene expression in the absence of stress.

While the 35S:ABF3 plants did not show any changes in transcription in the absence of drought stress, there were some phenotypic differences compared to control plants. Most notably, the 35S:ABF3 plants were smaller in size than control plants of the same age, with the difference becoming more pronounced with increased age (Figure 2B, C). This is a common observation for plants overexpressing transcription factors $[7,33,39,60]$ and is often overcome by using tissue-specific or inducible promoters $[32,60]$. At least some of the growth retardation may be attributable to reduced transpiration rates of $35 \mathrm{~S}: \mathrm{ABF} 3$ plants compared to control 
plants (Figure 2A), which is consistent with the observation that Arabidopsis plants overexpressing $A B F 3$ typically have stomata with smaller openings than do wild-type plants [7]. This would suggest that $A B F 3$ may govern gene networks involved in stomatal closure. Consistent with this, ABF3 is expressed in guard cells and its expression is further induced in these cells in response to ABA $[7,92]$. Since our analysis was performed on whole plants, it is likely that changes in the transcriptional network of guard cells would not be readily detectable.

\section{Overexpression of $A B F 3$ results in transcriptional reprogramming of the drought response}

Overexpression of $A B F 3$ confers drought tolerance to Arabidopsis plants and since ABF3 is a transcription factor, it can be predicted that this will occur through changes to the transcriptional network of the plants. Consistent with this, the expression profile of Arabidopsis plants overexpressing $A B F 3$ differed from that of control plants. As might be expected, there were a number of genes with expression patterns that appeared to be enhanced in 35S:ABF3 plants compared to control plants. Whether this occurred through alterations in the timing or strength of expression could not be established with the two time points considered in this study. Many of the genes with enhanced expression are known to function in mitigating drought stress, suggesting that they could contribute incrementally to the enhanced drought tolerance of 35S:ABF3 plants (Table 5). Those genes showing enhanced regulation in 35S:ABF3 plants likely include some direct targets of ABF3. In particular, those genes that are differentially expressed at $2 \mathrm{~h}$ and contain at least one ABRE are the most likely targets of ABF3 (Table 6).

Interestingly, there seemed to be a number of genes involved in RNA processing that showed enhanced expression in 35S:ABF3 plant lines. Most of these were downregulated at the $24 \mathrm{~h}$ time point. A number of RNA processing mutants impaired in ABA response have demonstrated the importance of RNA processing to ABA signalling [93]. Loss-of-function mutations in two genes encoding subunits of a nuclear cap-binding complex cause ABA hypersensitivity $[63,66]$ as do mutations in a pre-mRNA splicing factor that is important for both mRNA splicing and turnover [94], a poly(A)specific ribonuclease that is predicted to function in mRNA degradation [65], and a protein with homology to the Sm-like small nuclear ribonucleoproteins (snRNPs) that function in mRNA splicing, export, and degradation [95]. Two phosphatases that belong to the family of proteins that dephosphorylate the C-terminal domain of RNA polymerase II negatively regulate stress-responsive gene transcription [96] and a mutation in one of these results in decreased ABA sensitivity [97]. The enrichment of genes involved in RNA processing that show enhanced expression in the 35S:ABF3 line might suggest that ABF3 plays an important role in the regulation of ABA-responsive RNA processing events. The downregulation of many of the RNA processing genes is consistent with the negative regulatory role observed for several of the RNA processing proteins previously identified in the ABA-signalling pathways.

In addition to those genes showing enhanced regulation in 35S:ABF3 plants, there were also a number of genes with attenuated expression. Many of these are known to encode proteins with roles in minimizing drought stress and their attenuated expression is not consistent with the drought tolerance of the 35S:ABF3 plants. These genes may be reflective of a greater transcriptional reprogramming in 35S:ABF3 plants than merely enhancing the rate or level of expression of a subset of genes. The strong activation of the ABF3 pathway may result in co-ordinated feedback that modulates other drought responsive pathways, resulting in attenuated gene expression in some cases. This might reflect cooperativity between some of the drought signalling pathways. In many cases, droughtresponsive transcription factors have been shown to function in concert to activate gene expression $[39,40,43,90]$. Furthermore, it has been observed that downregulation of the phosphoinositide pathway in Arabidopsis results in an upregulation of the DREB2A gene as well as several DREB2A-regulated genes, suggesting a negative interaction between two drought signalling pathways [98].

The degree of stress experienced by the control plants should be greater than that experienced by the $35 \mathrm{~S}$ : ABF3 plants and this may ultimately have secondary consequences on the transcriptional network that is reflected by the genes showing an attenuated transcriptional response. This is consistent with the observation that several transposable element genes show attenuated expression in 35S:ABF3 plants (Table 9). Many transposons are activated in response to stress $[69,70]$ and their delayed activation in 35S:ABF3 plants could be a result of the drought tolerance of these plants.

Similarly, the enrichment of genes encoded by the chloroplast and mitochondrial genomes in the attenuated category (Table 8) may also reflect the greater drought sensitivity of the control plant lines. Drought has a significant and complex impact on the activities of both the mitochondria and chloroplasts and this is reflected in changes in gene expression $[99,100]$. Several of the chloroplast and mitochondria-encoded genes showing attenuated expression encode $\mathrm{NADH}$ dehydrogenases. The chloroplast NDH complex is 
predicted to function in cyclic electron transport around photosystem I to help dissipate excess energy during abiotic stresses such as drought and thereby to alleviate oxidative stress [67]. The elevated expression of chloroplast NDH complex subunits and other chloroplast and mitochondria-encoded genes in control plants may therefore be indicative of the increased stress of these plants.

Drought stress can change the composition of a plant, altering levels of oils, proteins and other constituents, which can affect the commercial and nutritional value of the plant [101-107]. In some cases, drought can also initiate the accumulation of higher levels of dangerous toxins and anti-nutrients $[102,105,108]$. The altered expression of transposable element genes and genes that are encoded by the chloroplast and mitochondrial genomes in control plants suggests that these plants are experiencing a higher level of drought stress than 35S: ABF3 plants. It is therefore possible that control plants exposed to drought will exhibit more compositional changes compared to unstressed plants than 35S:ABF3 plants. This is an important consideration because compositional analysis is one of the parameters used to determine the substantial equivalence of transgenic plants to their non-transgenic comparators during the risk assessment process. These results might suggest that 35S:ABF3 plants are better able to maintain compositional standards under drought stress than their nontransgenic counterparts.

\section{Overexpression of $A B F 3$ does not activate unintended gene networks during the drought response}

While differences were observed in the patterns of gene expression of 35S:ABF3 and control plant lines, at the functional level the response was very similar. Functional categorization of the significantly differentially expressed genes demonstrated that the percentage of genes in each category was relatively similar between the two plant lines (Additional file 2). This suggests that overexpression of $A B F 3$ does not activate new gene networks but simply functions to modify existing gene networks that function in drought response. Although there were genes that were uniquely regulated in each of the two plant lines, many of these genes did observe a change in expression in the other plant line that was not of a high enough magnitude to meet our criteria for differential expression (Additional file 3). This suggests that these genes are showing stronger and/or earlier differential regulation as opposed to being uniquely regulated.

Among the genes that were differentially regulated between the 35S:ABF3 and control plant lines, there was no indication that overexpression of $A B F 3$ activated any unintended gene networks. Several closely related members of the ABF/AREB transcription factor family function in seed germination and seed and early seedling developmental pathways, including ABI5 whose role in these pathways has been best characterized [109-111]. Although ABF3 is primarily associated with abiotic stress signalling in vegetative tissues, there is some evidence that it may also function in seed and early seedling developmental processes, although its role may be relatively minor. Microarray data from GENEVESTIGATOR [112] indicates that ABF3 is expressed during seed development, although at low levels compared to ABI5. Also, levels of $A B F 3$ are enhanced by dehydration and salinity stresses or by ABA treatment in germinating embryos [113] and an alternative splice form of the $A B F 3$ gene was identified from a cDNA library prepared from immature seed [91]. Double mutant analysis has also revealed several redundancies between ABF3 and ABI5, including sensitivity to ABA during germination, stress sensitivity of root growth, resistance to glucose, and regulation of the ABA-induced vegetative expression of $R A B 18$ and $R D 29 B$ [88]. $A B I 5$ and $A B F 3$ also appear to antagonistically cross-regulate each other $[88,114]$.

If ABF3 does function in seed developmental pathways, it is possible that altering its pattern of expression could ectopically activate gene networks involved in those pathways. Another member of the ABF/AREB family of transcription factors, ABF2/AREB1, which functions in abiotic stress signalling and glucose response, is also expressed in embryonic axes in dry siliques [33,77]. Overexpression of a phosphorylated active form of ABF2/AREB1 led to the activation of several seed storage protein genes in vegetative tissues, many of which also have binding sites for $\mathrm{ABI} 3$, another transcription factor involved in seed development [81]. However, none of the seed storage genes activated by the phosphorylated active form of $A B F 2 / A R E B 1$ were differentially expressed in the 35S:ABF3 plants, nor were AtEm1 and AtEm6, two LEA-class genes that are ABI5 targets expressed during seed maturation (data not shown). This further demonstrates that overexpression of $A B F 3$, while modifying patterns of gene expression, did not activate unintended pathways in response to drought stress in Arabidopsis. This suggests that overexpression of a transcription factor to confer an abiotic stress tolerance trait may not necessarily produce unintended pleiotropic effects.

\section{Conclusions}

The Cre/lox recombination system allowed us to create paired plant lines with identical T-DNA insertion sites either with or without the $A B F 3$ transgene in order to eliminate position effects in our analysis of unintended effects. This approach also allowed us to examine 
unintended effects resulting from the expression of Cre recombinase. We found that Cre recombinase had a minimal impact on the transcriptome, which suggests that it produces few unintended effects in Arabidopsis. Microarray analysis of Arabidopsis plants overexpressing $A B F 3$ demonstrated that the impact on the transcriptome is minimal. In the absence of drought stress, there were no differentially expressed genes. In response to drought stress, a reprogramming of the drought response was observed, suggestive of changes in the timing or strength of expression of some genes in 35S:ABF3 plants. Some of these changes may be directly related to the action of ABF3 while others may reflect an altered physiological state as a result of the enhanced drought tolerance of 35S:ABF3 plants. Amongst the differentially expressed genes, no unintended pathways appeared to be activated as a result of $A B F 3$ overexpression. These results are significant because they demonstrate that plant responses to abiotic stresses such as drought may be strictly coordinated at multiple regulatory steps and this limits the extent of unintended pleiotropic effects. This demonstrates that engineering stress tolerance through manipulation of endogenous plant pathways may not necessarily produce unintended pleiotropic effects despite the complexity of such traits. This is an important finding for establishing the safety of such traits as they begin to enter the market in the near future.

\section{Methods}

\section{Generation of transgenic plants}

To generate 35S:ABF3 plant lines, Arabidopsis thaliana Col-0 plants were transformed by the floral dip method [115] using Agrobacterium tumefaciens strain GV3101 harbouring the pCAMBIA3300 vector containing the Cauliflower mosaic virus $35 \mathrm{~S}$ promoter, $A B F 3$ coding region, and nopaline synthase transcriptional terminator, bordered on either side loxP sites. Homozygous single insert transformants were identified by Southern blot and segregation analysis and three lines with high levels of $A B F 3$ expression, as determined by RT-PCR analysis, were selected for further analysis.

To generate plant lines expressing $\mathrm{Cr}$ recombinase, Arabidopsis thaliana Col-0 plants were transformed as above with a pCAMBIA1200 vector containing the Cauliflower Mosaic Virus 35S promoter, Cre recombinase coding region, and nopaline synthase transcriptional terminator.

To generate control plant lines, 35S:ABF3 plants were crossed with plants expressing $\mathrm{Cre}$ recombinase in order to excise the 35S-ABF3-nos transgene. The F1 plants were PCR genotyped to identify those that underwent successful excision, using the primers ABF-F (5'ATGGgGTCTAGATTAAAC-3') and Nos-R (5'CCGATCTAGTAACATAGATG-3'), which are specific for the $A B F 3$ gene and the nopaline synthase transcriptional terminator, respectively. One plant from each cross, Control-48; Cre 1.1.2, Control-57; Cre 1.2.2 and Control-59; Cre 2.2.1, was selected from which loss of the 35S-ABF3-nos transgene was confirmed by PCR (Additional File 1). F2 progeny from the selected F1 plants were then backcrossed to wild-type plants to eliminate Cre recombinase. The F1 plants from this cross were again PCR genotyped to identify those from which the Cre gene was lost, using primers CRE.F (5'CCAGGCGTTTTCTGAGCATACCTG-3') and CRE.R (5'-CTCTGACCAGAGTCATCCTTAGCG-3'), which are both specific for the Cre recombinase gene. One plant from each cross, Control-48 1.1.2.5, Control-57 1.2.2.5 and Control-59 2.2.1.4, was selected from which loss of the Cre recombinase gene was confirmed by PCR (Additional File 1). The selected F1 plants represent the control plant lines and the F2 progeny of these plants were used in subsequent experiments.

\section{Growth of Arabidopsis plants}

Seeds were surface sterilized by soaking for $20 \mathrm{~min}$ in $25 \%(\mathrm{v} / \mathrm{v})$ commercial Clorox (final concentration of $1.3 \%$ sodium hypochlorite) and $0.05 \%(\mathrm{v} / \mathrm{v})$ Triton X100 (Fisher Scientific, Hampton, NH, USA), and then rinsing four times with distilled water. Seeds were germinated on MS media and grown at $22^{\circ} \mathrm{C}$ with a $16 \mathrm{~h}$ photoperiod. To determine the growth rate of plants, three-day-old seedlings were transplanted onto fresh MS plates and after one week or four weeks of growth the fresh weight of the seedlings was measured. To measure transpiration rate, leaves of a similar developmental stage were excised from four-week-old plants and the loss of weight over a 24 h-period was measured. For microarray analysis, seeds were germinated on MS media and one-week-old seedlings were collected. For the drought stress, seedlings were transferred onto paper towels and harvested after 2 and $24 \mathrm{~h}$.

\section{Microarray Analysis}

For each sample, three biological replicates were prepared. RNA was extracted from seedlings using the RNeasy Plant Mini Kit (Qiagen, Germantown, MD, USA), following the manufacturer's protocol. A 2100 Bioanalyser (Agilent Technologies, Santa Clara, CA, USA) was used to determine the quality of the RNA. Microarray analysis was performed using the GeneChip ${ }^{\circ}$ Arabidopsis ATH1 Genome Array (Affymetrix Inc., Santa Clara, CA, USA). Standard RNA processing, hybridization, and scanning protocols were followed as recommended by the GeneChip Expression Analysis Technical Manual (Affymetrix Inc., Santa Clara, CA, USA). Hybridization, and scanning were performed at Agriculture and Agri-Food Canada (Winnipeg, MB, 
Canada) by Mark Jordan. RMA procedure [116] was performed to normalize data using the AffylmGUI R software package from Bioconductor (http://www.bioconductor.org/)[117]. Analysis of differential expression was done using a moderated t-test with empirical Bayes smoothing [118]. Microarray data from this study have been deposited at ArrayExpress (accession number EMEXP-2435).

\section{Reverse transcription-PCR (RT-PCR)}

DNaseI-treated RNA was used for first strand cDNA synthesis using Superscript III reverse transcriptase (Invitrogen, Carlsbad, CA, USA) and oligo $(\mathrm{dT})_{18}$ primers according to the manufacturer's protocol. Between 24 and 30 cycles of PCR amplification was performed using genespecific primers. As an internal control, elongation factor $1-\alpha(E F-1)$ was amplified. Sequences of all primers used in RT-PCR analysis can be found in Additional file 4.

\section{List of Abbreviations}

ABF: ABA-responsive element binding factor; AREB: ABA-responsive element binding protein; $A B A$ : abscisic acid; ABRE: ABA-responsive element; DREB: dehydration responsive element binding protein; $\mathrm{CBF}$ : $\mathrm{C}$-repeat binding factor; NAC: NAM, ATAF1,2, CUC2; ZFHD: zinc finger homeodomain; CaMV 35 S promoter: Cauliflower Mosaic Virus 35S promoter; RT-PCR: reverse transcription polymerase chain reaction; LEA proteins: late embryogenesis abundant proteins; NDH complex: NADH dehydrogenase complex; ABI: ABA-insensitive.

Additional file 1: PCR genotyping of control plant lines. (A) Control plant lines, derived from 35S:ABF3 plants crossed with plants expressing Cre recombinase, were PCR genotyped to identify plants from which the 35S-ABF3-nos transgene was excised. (B) Control plant lines following a backcross with Col-0 plants were genotyped to identify plants that lost the Cre gene by genetic segregation. Plant lines with the desired genotype that were selected following each cross are indicated with asterisks.

Click here for file

[http://www.biomedcentral.com/content/supplementary/1471-2164-1169-S1.PDF ]

Additional file 2: Functional categorization of drought responsive genes in 35S:ABF3 and control plants. Distribution of genes differentially expressed in 35S:ABF3 and control plant lines at $2 \mathrm{~h}$ and 24 $\mathrm{h}$ following drought stress into functional categories.

Click here for file

[http://www.biomedcentral.com/content/supplementary/1471-2164-1169-S2.TIFF ]

Additional file 3: Genes differentially expressed in response to drought in 35S:ABF3 and control plants. This table includes a complete list of genes differentially expressed in both 35S:ABF3 and control plant lines in response to drought at $2 \mathrm{~h}$ and $24 \mathrm{~h}$. Genes are divided into three groups including those that are similarly regulated in both 35S:ABF3 and control plants, those that show enhanced regulation in 35S:ABF3 plants, and those that show attenuated regulation in 35S: ABF3 plants.

Click here for file

[http://www.biomedcentral.com/content/supplementary/1471-2164-1169-S3.XLS]
Additional file 4: Sequences of primers used in RT-PCR analysis. This table includes sequences of primers used to amplify selected genes in the RT-PCR analysis performed to confirm microarray results.

Click here for file

[http://www.biomedcentral.com/content/supplementary/1471-2164-1169-S4.XLS ]

\section{Acknowledgements}

We would like to thank Hélène Labbé for her assistance in preparing RNA samples for microarray hybridization. Probe synthesis, hybridization, and scanning were performed by Mark Jordan at Agriculture and Agri-Food Canada (Winnipeg, MB, Canada). The gene encoding Cre recombinase was provided by Teresa Martin. This research was supported by funding from the Plant Biosafety Office, Feeds Section, and the Plant and Biotechnology Risk Assessment Unit of the Canadian Food Inspection Agency. Both AA and JS were supported by Visiting Fellowships in Canadian Government Laboratories granted by NSERC.

\section{Author details}

${ }^{1}$ Agriculture and Agri-Food Canada, 960 Carling Ave., Ottawa, ON, K1A OC6 Canada. ${ }^{2}$ Current address: Department of Biology, McGill University, 1205 Docteur Penfield Ave., Montreal, QC, H3A 1B1 Canada. ${ }^{3}$ Current address: Plant and Biotechnology Risk Assessment Unit, Canadian Food Inspection Agency, 1400 Merivale Rd., Ottawa, ON, K1A OY9 Canada.

\section{Authors' contributions}

AA participated in the design of the study and carried out the vector construction, plant transformation and molecular analysis, microarray analysis, and contributed to the drafting of the manuscript. JS participated in the final microarray analysis, carried out the RT-PCR work, and drafted the manuscript. BM conceived of the study and participated in its design and coordination, and participated in the drafting of the manuscript. All authors read and approved the final manuscript.

Received: 12 August 2009

Accepted: 28 January 2010 Published: 28 January 2010

\section{References}

1. Boyer JS: Plant productivity and environment. Science 1982, 218:443-448.

2. Burke EJ, Brown SJ, Christidis N: Modeling the recent evolution of global drought and projections for the twenty-first century with the Hadley Centre climate model. J Hydrometeorology 2006, 7:1113-1125.

3. Meehl GA, Washington WM, Santer BD, Collins WD, Arblaster JM, Hu A, Lawrence DM, Teng H, Buja LE, Strand WG: Climate change projections for the twenty-first century and climate change commitment in the CCSM3. J Climate 2006, 19:2597-2616.

4. Century K, Reuber TL, Ratcliffe OJ: Regulating the regulators: the future prospects for transcription-factor-based agricultural biotechnology products. Plant Physiol 2008, 147:20-29.

5. Salmeron J, Herrera-Estrella LR: Plant biotechnology: Fast-forward genomics for improved crop production. Curr Opin Plant Biol 2006, 9:177-179.

6. Ding Z, Li S, An X, Liu X, Qin H, Wang D: Transgenic expression of MYB15 confers enhanced sensitivity to abscisic acid and improved drought tolerance in Arabidopsis thaliana. J Genet Genomics 2009, 36:17-29.

7. Kang JY, Choi HI, Im MY, Kim SY: Arabidopsis basic leucine zipper proteins that mediate stress-responsive abscisic acid signaling. Plant Cell 2002, 14:343-357.

8. Liu Q, Kasuga M, Sakuma Y, Abe H, Miura S, Yamaguchi-Shinozaki K, Shinozaki K: Two transcription factors, DREB1 and DREB2, with an EREBP/ AP2 DNA binding domain separate two cellular signal transduction pathways in drought- and low-temperature-responsive gene expression, respectively, in Arabidopsis. Plant Cell 1998, 10:1391-1406.

9. Sakuma Y, Maruyama K, Osakabe Y, Qin F, Seki M, Shinozaki K, YamaguchiShinozaki K: Functional analysis of an Arabidopsis transcription factor, DREB2A, involved in drought-responsive gene expression. Plant Cell 2006, 18:1292-1309. 
10. Hu H, Dai M, Yao J, Xiao B, Li X, Zhang Q, Xiong L: Overexpressing a NAM, ATAF, and CUC (NAC) transcription factor enhances drought resistance and salt tolerance in rice. Proc Natl Acad Sci USA 2006, 103:12987-12992.

11. Oh SJ, Song SI, Kim YS, Jang HJ, Kim SY, Kim M, Kim YK, Nahm BH, Kim JK. Arabidopsis $\mathrm{CBF} 3 / \mathrm{DREB} 1 \mathrm{~A}$ and $\mathrm{ABF} 3$ in transgenic rice increased tolerance to abiotic stress without stunting growth. Plant Physiol 2005 138:341-351.

12. Yi K, Wu Z, Zhou J, Du L, Guo L, Wu Y, Wu P: OsPTF1, a novel transcription factor involved in tolerance to phosphate starvation in rice. Plant Physiol 2005, 138:2087-2096.

13. Nelson DE, Repetti PP, Adams TR, Creelman RA, Wu J, Warner DC Anstrom DC, Bensen RJ, Castiglioni PP, Donnarummo MG, et al: Plant nuclear factor $Y$ (NF-Y) B subunits confer drought tolerance and lead to improved corn yields on water-limited acres. Proc Natl Acad Sci USA 2007, 104:16450-16455.

14. Winicov I, Bastola DR: Transgenic overexpression of the transcription factor alfin1 enhances expression of the endogenous MsPRP2 gene in alfalfa and improves salinity tolerance of the plants. Plant Physiol 1999, 120:473-480

15. Miki B, Abdeen A, Manabe $Y$, MacDonald P: Selectable marker genes and unintended changes to the plant transcriptome. Plant Biotechnol J 2009, 7:211-218.

16. Eulgem T, Rushton PJ, Robatzek S, Somssich IE: The WRKY superfamily of plant transcription factors. Trends Plant Sci 2000, 5:199-206.

17. Rijpkema AS, Gerats T, Vandenbussche M: Evolutionary complexity of MADS complexes. Curr Opin Plant Biol 2007, 10:32-38.

18. Abdeen A, Miki B: The pleiotropic effects of the bar gene and glufosinate on the Arabidopsis transcriptome. Plant Biotechnol J 2009, 7:266-282.

19. Baker JM, Hawkins ND, Ward JL, Lovegrove A, Napier JA, Shewry PR, Beale $\mathrm{MH}$ : A metabolomic study of substantial equivalence of fieldgrown genetically modified wheat. Plant Biotechnol J 2006, 4:381-392.

20. Baudo MM, Lyons R, Powers S, Pastori GM, Edwards KJ, Holdsworth MJ, Shewry PR: Transgenesis has less impact on the transcriptome of wheat grain than conventional breeding. Plant Biotechnol J 2006, 4:369-380.

21. Albo AG, Mila S, Digilio G, Motto M, Aime S, Corpillo D: Proteomic analysis of a genetically modified maize flour carrying $C R Y 1 A B$ gene and comparison to the corresponding wild-type. Maydica 2007, 52:443-455.

22. Catchpole GS, Beckmann M, Enot DP, Mondhe M, Zywicki B, Taylor J, Hardy N, Smith A, King RD, Kell DB, et al: Hierarchical metabolomics demonstrates substantial compositional similarity between genetically modified and conventional potato crops. Proc Natl Acad Sci USA 2005, 102:14458-14462.

23. Cheng KC, Beaulieu J, lquira E, Belzile FJ, Fortin MG, Strömvik MV: Effect of transgenes on global gene expression in soybean is within the natural range of variation of conventional cultivars. J Agric Food Chem 2008, 56:3057-3067.

24. Corpillo D, Gardini G, Vaira AM, Basso M, Aime S, Accotto GP, Fasano M: Proteomics as a tool to improve investigation of substantial equivalence in genetically modified organisms: The case of a virus-resistant tomato. Proteomics 2004, 4:193-200.

25. El Ouakfaoui S, Miki B: The stability of the Arabidopsis transcriptome in transgenic plants expressing the marker genes nptll and uidA. Plant $J$ 2005, 41:791-800.

26. Gregersen PL, Brinch-Pedersen H, Holm PB: A microarray-based comparative analysis of gene expression profiles during grain development in transgenic and wild type wheat. Transgenic Res 2005, 14:887-905.

27. Lehesranta SJ, Davies HV, Shepherd LVT, Nunan N, McNicol JW, Auriola S, Koistinen KM, Suomalainen S, Kokko HI, Kärenlampi SO: Comparison of tuber proteomes of potato varieties, landraces, and genetically modified lines. Plant Physiol 2005, 138:1690-1699.

28. Jakoby M, Weisshaar B, Dröge-Laser W, Vicente-Carbajosa J, Tiedemann J, Kroj T, Parcy F: bZIP transcription factors in Arabidopsis. Trends Plant Sci 2002, 7:106-111.

29. Choi $H$, Hong J, Ha J, Kang J, Kim SY: ABFs, a family of ABA-responsive element binding factors. J Biol Chem 2000, 275:1723-1730.

30. Shinozaki K, Yamaguchi-Shinozaki K: Gene networks involved in drought stress response and tolerance. J Exp Bot 2007, 58:221-227.

31. Uno Y, Furihata $T$, Abe H, Yoshida R, Shinozaki K, Yamaguchi-Shinozaki K Arabidopsis basic leucine zipper transcription factors involved in an abscisic acid-dependent signal transduction pathway under drought and high-salinity conditions. Proc Natl Acad Sci USA 2000, 97:11632-11637.

32. Kim JB, Kang JY, Kim SY: Over-expression of a transcription factor

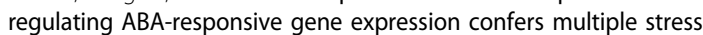
tolerance. Plant Biotechnol J 2004, 2:459-466.

33. Kim S, Kang JY, Cho DI, Park JH, Kim SY: ABF2, an ABRE-binding bZIP factor, is an essential component of glucose signaling and its overexpression affects multiple stress tolerance. Plant J 2004, 40:75-87.

34. Seki M, Narusaka M, Ishida J, Nanjo T, Fujita M, Oono $Y$, Kamiya A, Nakajima M, Enju A, Sakurai T, et al: Monitoring the expression profiles of 7000 Arabidopsis genes under drought, cold and high-salinity stresses using a full-length cDNA microarray. Plant J 2002, 31:279-292.

35. Seki M, Narusaka M, Abe H, Kasuga M, Yamaguchi-Shinozaki K, Carninci $P$, Hayashizaki Y, Shinozaki K: Monitoring the expression pattern of 1300 Arabidopsis genes under drought and cold stresses by using a fulllength cDNA microarray. Plant Cell 2001, 13:61-72.

36. Matsui A, Ishida J, Morosawa T, Mochizuki Y, Kaminuma E, Endo TA, Okamoto M, Nambara E, Nakajima M, Kawashima M, et al: Arabidopsis transcriptome analysis under drought, cold, high-salinity and ABA treatment conditions using a tiling array. Plant Cell Physiol 2008, 49:1135-1149.

37. Nakashima K, Shinwari ZK, Sakuma Y, Seki M, Miura S, Shinozaki K, Yamaguchi-Shinozaki K: Organization and expression of two Arabidopsis DREB2 genes encoding DRE-binding proteins involved in dehydrationand high-salinity-responsive gene expression. Plant Mol Biol 2000, 42:657-665.

38. Haake V, Cook D, Riechmann JL, Pineda O, Thomashow MF, Zhang JZ: Transcription factor CBF4 is a regulator of drought adaptation in Arabidopsis. Plant Physiol 2002, 130:639-648.

39. Abe H, Urao T, Ito T, Seki M, Shinozaki K, Yamaguchi-Shinozaki K: Arabidopsis AtMYC2 (bHLH) and AtMYB2 (MYB) function as transcriptional activators in abscisic acid signaling. Plant Cell 2003, 15:63-78.

40. Abe H, Yamaguchi-Shinozaki K, Urao T, Iwasaki T, Hosokawa D, Shinozaki K Role of arabidopsis MYC and MYB homologs in drought- and abscisic acid-regulated gene expression. Plant Cell 1997, 9:1859-1868.

41. Urao T, Yamaguchi-Shinozaki K, Urao S, Shinozaki K: An Arabidopsis myb homolog is induced by dehydration stress and its gene product binds to the conserved MYB recognition sequence. Plant Cell 1993, 5:1529-1539.

42. Tran LSP, Nakashima K, Sakuma Y, Simpson SD, Fujita Y, Maruyama K, Fujita M, Seki M, Shinozaki K, Yamaguchi-Shinozaki K: Isolation and functional analysis of Arabidopsis stress-inducible NAC transcription factors that bind to a drought-responsive cis-element in the early responsive to dehydration stress 1 promoter. Plant Cell 2004, 16:2481-2498.

43. Tran LSP, Nakashima K, Sakuma Y, Osakabe Y, Oin F, Simpson SD, Maruyama K, Fujita Y, Shinozaki K, Yamaguchi-Shinozaki K: Co-expression of the stress-inducible zinc finger homeodomain ZFHD1 and NAC transcription factors enhances expression of the ERD1 gene in Arabidopsis. Plant J 2006, 49:46-63.

44. Gilbertson L: Cre-lox recombination: Cre-ative tools for plant biotechnology. Trends Biotechnol 2003, 21:550-555.

45. De Buck S, Peck I, De Wilde C, Marjanac G, Nolf J, De Paepe A, Depicker A Generation of single-copy T-DNA transformants in Arabidopsis by the CRE/loxP recombination-mediated resolution system. Plant Physiol 2007, 145:1171-1182.

46. Hoa TTC, Bong BB, Huq E, Hodges TK: Cre/lox site-specific recombination controls the excision of a transgene from the rice genome. Theor Appl Genet 2002, 104:518-525.

47. Osborne Bl, Wirtz U, Baker B: A system for insertional mutagenesis and chromosomal rearrangement using the Ds transposon and Cre-lox. Plant J 1995, 7:687-701.

48. Russell $\mathrm{SH}$, Hoopes $J \mathrm{~L}$, Odell JT: Directed excision of a transgene from the plant genome. Mol Genet Genet 1992, 234:49-59.

49. Coppoolse ER, de Vroomen MJ, Roelofs D, Smit J, van Gennip F, Hersmus BJM, Nijkamp HJJ, van Haaren MJJ: Cre recombinase expression can result in phenotypic aberrations in plants. Plant Mol Biol 2003, 51:263-279.

50. Que Q, Wang HY, Jorgensen RA: Distinct patterns of pigment suppression are produced by allelic sense and antisense chalcone synthase transgenes in petunia flowers. Plant J 1998, 13:401-409. 
51. Corneille S, Lutz K, Svab Z, Maliga P: Efficient elimination of selectable marker genes from the plastid genome by the CRE-lox site-specific recombination system. Plant J 2001, 27:171-178.

52. Corneille S, Lutz KA, Azhagiri AK, Maliga P: Identification of functional lox sites in the plastid genome. Plant J 2003, 35:753-762.

53. Hajdukiewicz PTJ, Gilbertson L, Staub JM: Multiple pathways for Cre/loxmediated recombination in plastids. Plant J 2001, 27:161-170.

54. Heidmann D, Lehner CF: Reduction of Cre recombinase toxicity in proliferating Drosophila cells by estrogen-dependent activity regulation. Dev Genes Evol 2001, 211:458-465.

55. Loonstra A, Vooijs M, Beverloo HB, Allak BA, van Drunen E, Kanaar R, Berns A, Jonkers J: Growth inhibition and DNA damage induced by Cre recombinase in mammalian cells. Proc Natl Acad Sci USA 2001, 98:9209-9214.

56. Schmidt EE, Taylor DS, Prigge JR, Barnett S, Capecchi MR: Illegitimate Credependent chromosome rearrangements in transgenic mouse spermatids. Proc Natl Acad Sci USA 2000, 97:13702-13707.

57. Silver DP, Livingston DM: Self-excising retroviral vectors encoding the Cre recombinase overcome Cre-mediated cellular toxicity. Mol Cell 2001, 8:233-243.

58. Li J, Brader G, Palva ET: The WRKY70 Transcription Factor: A Node of Convergence for Jasmonate-Mediated and Salicylate-Mediated Signals in Plant Defense. Plant Cell 2004, 16:319-331.

59. Agarwal PK, Agarwal P, Reddy MK, Sopory SK: Role of DREB transcription factors in abiotic and biotic stress tolerance in plants. Plant Cell Rep 2006, 25:1263-1274.

60. Kasuga M, Liu Q, Miura S, Yamaguchi-Shinozaki K, Shinozaki K: Improving plant drought, salt, and freezing tolerance by gene transfer of a single stress-inducible transcription factor. Nat Biotechnol 1999, 17:287-291.

61. Novillo F, Alonso JM, Ecker JR, Salinas J: CBF2/DREB1C is a negative regulator of $C B F 1 / D R E B 1 B$ and $C B F 3 / D R E B 1 A$ expression and plays a central role in stress tolerance in Arabidopsis. Proc Natl Acad Sci USA 2004, 101:3985-3990

62. Gómez-Porras JL, Riaño-Pachón DM, Dreyer I, Mayer JE, Mueller-Roeber B: Genome-wide analysis of ABA-responsive elements ABRE and CE3 reveals divergent patterns in Arabidopsis and rice. BMC Genomics 2007 $8: 260$

63. Hugouvieux V, Kwak JM, Schroeder Jl: An mRNA cap binding protein, $\mathrm{ABH} 1$, modulates early abscisic acid signal transduction in Arabidopsis. Cell 2001, 106:477-487.

64. Lu C, Fedoroff N: A mutation in the Arabidopsis HYL1 gene encoding a dsRNA binding protein affects responses to abscisic acid, auxin, and cytokinin. Plant Cell 2000, 12:2351-2365

65. Nishimura N, Kitahata N, Seki M, Narusaka Y, Narusaka M, Kuromori T, Asami T, Shinozaki K, Hirayama T: Analysis of ABA hypersensitive germination2 revealed the pivotal functions of PARN in stress response in Arabidopsis. Plant J 2005, 44:972-984.

66. Papp I, Mur LA, Dalmadi A, Dulai S, Koncz C: A mutation in the Cap Binding Protein 20 gene confers drought tolerance to Arabidopsis. Plant Mol Biol 2004, 55:679-686.

67. Rumeau D, Peltier G, Cournac L: Chlororespiration and cyclic electron flow around PSI during photosynthesis and plant stress response. Plant Cell Environ 2007, 30:1041-1051.

68. Casano LM, Zapata JM, Martín M, Sabater B: Chlororespiration and poising of cyclic electron transport. Plastoquinone as electron transporter between thylakoid NADH dehydrogenase and peroxidase. J Biol Chem 2000, 275:942-948

69. Grandbastien M-A: Activation of plant retrotransposons under stress conditions. Trends Plant Sci 1998, 3:181-187.

70. Madlung A, Comai L: The effect of stress on genome regulation and structure. Ann Bot 2004, 94:481-495.

71. Sauer B: Identification of cryptic lox sites in the yeast genome by selection for Cre-mediated chromosome translocations that confer multiple drug resistance. J Mol Biol 1992, 223:911-928.

72. Thyagarajan B, Guimarães MJ, Groth AC, Calos MP: Mammalian genomes contain active recombinase recognition sites. Gene 2000, 244:47-54.

73. Drescher A, Ruf S, Calsa T Jr, Carrer H, Bock R: The two largest chloroplast genome-encoded open reading frames of higher plants are essential genes. Plant J 2000, 22:97-104.

74. Shikanai T, Shimizu K, Ueda K, Nishimura Y, Kuroiwa T, Hashimoto T: The chloroplast ClpP gene, encoding a proteolytic subunit of ATP-dependent protease, is indispensable for chloroplast development in tobacco. Plant Cell Physiol 2001, 42:264-273.

75. Legen J, Wanner G, Herrmann RG, Small I, Schmitz-Linneweber C: Plastid tRNA genes trnC-GCA and trnN-GUU are essential for plant cell development. Plant J 2007, 51:751-762.

76. Bensmihen S, Giraudat J, Parcy F: Characterization of three homologous basic leucine zipper transcription factors (bZIP) of the ABI5 family during Arabidopsis thaliana embryo maturation. J Exp Bot 2005, 56:597-603.

77. Fujita Y, Fujita M, Satoh R, Maruyama K, Parvez MM, Seki M, Hiratsu K, Ohme-Takagi M, Shinozaki K, Yamaguchi-Shinozaki K: AREB1 is a transcription activator of novel ABRE-dependent ABA signaling that enhances drought stress tolerance in Arabidopsis. Plant Cell 2005, 17:3470-3488

78. Lopez-Molina L, Mongrand S, Chua NH: A postgermination developmental arrest checkpoint is mediated by abscisic acid and requires the $A B I 5$ transcription factor in Arabidopsis. Proc Natl Acad Sci USA 2001, 98:4782-4787.

79. Chae MJ, Lee JS, Nam MH, Cho K, Hong JY, Yi SA, Suh SC, Yoon IS: A rice dehydration-inducible SNF1-related protein kinase 2 phosphorylates an abscisic acid responsive element-binding factor and associates with $A B A$ signaling. Plant Mol Biol 2007, 63:151-169.

80. Fujii $H$, Verslues PE, Zhu JK: Identification of two protein kinases required for abscisic acid regulation of seed germination, root growth, and gene expression in Arabidopsis. Plant Cell 2007, 19:485-494.

81. Furihata T, Maruyama K, Fujita Y, Umezawa T, Yoshida R, Shinozaki K, Yamaguchi-Shinozaki K: Abscisic acid-dependent multisite phosphorylation regulates the activity of a transcription activator AREB1. Proc Natl Acad Sci USA 2006, 103:1988-1993.

82. Johnson RR, Wagner RL, Verhey SD, Walker-Simmons MK: The abscisic acidresponsive kinase PKABA1 interacts with a seed-specific abscisic acid response element-binding factor, TaABF, and phosphorylates TaABF peptide sequences. Plant Physiol 2002, 130:837-846.

83. Kagaya Y, Hobo T, Murata M, Ban A, Hattori T: Abscisic acid-induced transcription is mediated by phosphorylation of an abscisic acid response element binding factor, TRAB1. Plant Cell 2002, 14:3177-3189.

84. Kobayashi $Y$, Murata M, Minami H, Yamamoto S, Kagaya Y, Hobo T, Yamamoto A, Hattori T: Abscisic acid-activated SNRK2 protein kinases function in the gene-regulation pathway of $A B A$ signal transduction by phosphorylating ABA response element-binding factors. Plant J 2005, 44:939-949.

85. Choi H, Park HJ, Park JH, Kim S, Im MY, Seo HH, Kim YW, Hwang I, Kim SY: Arabidopsis calcium-dependent protein kinase AtCPK32 interacts with ABF4, a transcriptional regulator of abscisic acid-responsive gene expression, and modulates its activity. Plant Physiol 2005, 139:1750-1761.

86. Zhu SY, Yu XC, Wang XJ, Zhao R, Li Y, Fan RC, Shang Y, Du SY, Wang XF, Wu FQ, et al: Two calcium-dependent protein kinases, CPK4 and CPK11, regulate abscisic acid signal transduction in Arabidopsis. Plant Cell 2007 19:3019-3036.

87. Kim S, Choi H, Ryu HJ, Park JH, Kim MD, Kim SY: ARIA, an Arabidopsis arm repeat protein interacting with a transcriptional regulator of abscisic acid-responsive gene expression, is a novel abscisic acid signaling component. Plant Physiol 2004, 136:3639-3648.

88. Finkelstein R, Gampala SSL, Lynch TJ, Thomas TL, Rock CD: Redundant and distinct functions of the ABA response loci ABA-INSENSITIVE(ABI)5 and ABRE-BINDING FACTOR (ABF)3. Plant Mol Biol 2005, 59:253-267.

89. Nakamura S, Lynch TJ, Finkelstein RR: Physical interactions between ABA response loci of Arabidopsis. Plant J 2001, 26:627-635.

90. Narusaka Y, Nakashima K, Shinwari ZK, Sakuma Y, Furihata T, Abe H, Narusaka M, Shinozaki K, Yamaguchi-Shinozaki K: Interaction between two cis-acting elements, ABRE and DRE, in ABA-dependent expression of Arabidopsis $r d 29 A$ gene in response to dehydration and high-salinity stresses. Plant J 2003, 34:137-148.

91. Kim SY, Ma J, Perret P, Li Z, Thomas TL: Arabidopsis ABI5 subfamily members have distinct DNA-binding and transcriptional activities. Plant Physiol 2002, 130:688-697.

92. Yang Y, Costa A, Leonhardt N, Siegel RS, Schroeder Jl: Isolation of a strong Arabidopsis guard cell promoter and its potential as a research tool. Plant Methods 2008, 4:6.

93. Kuhn JM, Schroeder J: Impacts of altered RNA metabolism on abscisic acid signaling. Curr Opin Plant Biol 2003, 6:463-469. 
94. Lee B, Kapoor A, Zhu J, Zhu JK: STABILIZED1, a stress-upregulated nuclear protein, is required for pre-mRNA splicing, mRNA turnover, and stress tolerance in Arabidopsis. Plant Cell 2006, 18:1736-1749.

95. Xiong L, Gong Z, Rock CD, Subramanian S, Guo Y, Xu W, Galbraith D, Zhu JK: Modulation of abscisic acid signal transduction and biosynthesis by an Sm-like protein in Arabidopsis. Dev Cell 2001, 1:771-781.

96. Koiwa H, Barb AW, Xiong L, Li F, McCully MG, Lee BH, Sokolchik I, Zhu J, Gong Z, Reddy M, et al: C-terminal domain phosphatase-like family members (AtCPLs) differentially regulate Arabidopsis thaliana abiotic stress signaling, growth, and development. Proc Natl Acad Sci USA 2002, 99:10893-10898.

97. Xiong L, Lee $H$, Ishitani M, Tanaka Y, Stevenson B, Koiwa H, Bressan RA, Hasegawa PM, Zhu JK: Repression of stress-responsive genes by FIERY2, a novel transcriptional regulator in Arabidopsis. Proc Natl Acad Sci USA 2002, 99:10899-10904.

98. Perera IY, Hung CY, Moore CD, Stevenson-Paulik J, Boss WF: Transgenic Arabidopsis plants expressing the type 1 inositol 5-phosphatase exhibit increased drought tolerance and altered abscisic acid signaling. Plant Cell 2008, 20:2876-2893.

99. Atkin OK, Macherel D: The crucial role of plant mitochondria in orchestrating drought tolerance. Ann Bot 2009, 103:581-597.

100. Chaves MM, Flexas J, Pinheiro C: Photosynthesis under drought and salt stress: regulation mechanisms from whole plant to cell. Ann Bot 2009 103:551-560.

101. Carvalho IS, Chaves MM, Pinto Ricardo C: Influence of Water Stress on the Chemical Composition of Seeds of Two Lupins (Lupinus albus and Lupinus mutabilis). J Agron Crop Sci 2005, 191:95-98.

102. Champolivier L, Merrien A: Effects of water stress applied at different growth stages to Brassica napus L. var. oleifera on yield, yield components and seed quality. Eur J Agron 1996, 5:153-160.

103. Dornbos DL Jr, Mullen RE: Soybean seed protein and oil contents and fatty acid composition adjustments by drought and temperature. J Am Oil Chem Soc 1992, 69:228-231.

104. Gigon A, Matos AR, Laffray D, Zuily-Fodil Y, Pham-Thi AT: Effect of drought stress on lipid metabolism in the leaves of Arabidopsis thaliana (ecotype Columbia). Ann Bot 2004, 94:345-351.

105. Jensen CR, Mogensen VO, Mortensen G, Fieldsend JK, Milford GFJ, Andersen MN, Thage JH: Seed glucosinolate, oil and protein contents of field-grown rape (Brassica napus L.) affected by soil drying and evaporative demand. Field Crops Res 1996, 47:93-105.

106. Rotundo $J$, Westgate ME: Meta-analysis of environmental effects on soybean seed composition. Field Crops Res 2009, 110:147-156.

107. Dakhma WS, Zarrouk M, Cherif A: Effects of drought-stress on lipids in rape leaves. Phytochemistry 1995, 40:1383-1386.

108. Bejarano L, Mignolet E, Devaux A, Espinola N, Carrasco E, Larondelle Y: Glycoalkaloids in potato tubers: the effect of variety and drought stress on the $\alpha$-solanine and $\alpha$-chaconine contents of potatoes. J Sci Food Agric 2000, 80:2096-2100.

109. Finkelstein RR, Lynch TJ: The Arabidopsis abscisic acid response gene $A B 15$ encodes a basic leucine zipper transcription factor. Plant Cell 2000, 12:599-610.

110. Lopez-Molina L, Mongrand S, McLachlin DT, Chait BT, Chua NH: ABI5 acts downstream of $A B \mid 3$ to execute an $A B A$-dependent growth arrest during germination. Plant J 2002, 32:317-328.

111. Carles C, Bies-Etheve N, Aspart L, Léon-Kloosterziel KM, Koornneef M, Echeverria M, Delseny M: Regulation of Arabidopsis thaliana Em genes: role of ABI5. The Plant Journal 2002, 30:373-383.

112. Zimmermann P, Hirsch-Hoffmann M, Hennig L, Gruissem W: GENEVESTIGATOR. Arabidopsis microarray database and analysis toolbox. Plant Physiol 2004, 136:2621-2632.

113. Nakashima K, Fujita Y, Katsura K, Maruyama K, Narusaka Y, Seki M, Shinozaki K, Yamaguchi-Shinozaki K: Transcriptional regulation of ABI3and ABA-responsive genes including RD29B and RD29A in seeds, germinating embryos, and seedlings of Arabidopsis. Plant Mol Biol 2006, 60:51-68.

114. Brocard IM, Lynch TJ, Finkelstein RR: Regulation and role of the Arabidopsis abscisic acid-insensitive 5 gene in abscisic acid, sugar, and stress response. Plant Physiol 2002, 129:1533-1543.

115. Clough SJ, Bent AF: Floral dip: a simplified method for Agrobacteriummediated transformation of Arabidopsis thaliana. Plant J 1998, 16:735-743.
116. Irizarry RA, Hobbs B, Collin F, Beazer-Barclay YD, Antonellis KJ, Scherf U, Speed TP: Exploration, normalization, and summaries of high density oligonucleotide array probe level data. Biostatistics 2003, 4:249-264.

117. Gentleman RC, Carey VJ, Bates DM, Bolstad B, Dettling M, Dudoit S, Ellis B, Gautier L, Ge Y, Gentry J, et al: Bioconductor: open software development for computational biology and bioinformatics. Genome Biol 2004, 5:R80.

118. Smyth GK: Linear models and empirical Bayes methods for assessing differential expression in microarray experiments. Stat Appl Genet Mol Biol 2004, 3, Article 3.

doi:10.1186/1471-2164-11-69

Cite this article as: Abdeen et al:: Transcriptome analysis reveals absence of unintended effects in drought-tolerant transgenic plants overexpressing the transcription factor ABF3. BMC Genomics 2010 11:69.

\section{Submit your next manuscript to BioMed Central and take full advantage of:}

- Convenient online submission

- Thorough peer review

- No space constraints or color figure charges

- Immediate publication on acceptance

- Inclusion in PubMed, CAS, Scopus and Google Scholar

- Research which is freely available for redistribution

Submit your manuscript at www.biomedcentral.com/submit
Biomed Central 